\title{
Morphometric variability in the diatom Fragilariopsis kerguelensis: Implications for Southern Ocean paleoceanography
}

\author{
G. Cortese *, R. Gersonde ${ }^{1}$ \\ Alfred Wegener Institute for Polar and Marine Research, P.O. Box 120161 - 27515 Bremerhaven, Germany
}

Received 7 September 2006; received in revised form 31 January 2007; accepted 6 March 2007

Available online 16 March 2007

Editor: M.L. Delaney

\begin{abstract}
Diatoms play a central role in the ecosystem of the Southern Ocean, where they represent the main producers and carriers of organic carbon and dissolved silicon towards the deep ocean. Variability in space and time of the size of the most abundant species (Fragilariopsis kerguelensis) may directly impact the way this ecosystem functions, and also affect the nutrient balance of the global ocean, as important water masses (e.g. the Antarctic Intermediate Water) get their nutrient signature in this area. We used a biometric approach and analyzed the size variability of $F$. kerguelensis valves in sixty-four surface sediment samples from the Southern Ocean, in sediment trap samples from a mooring (Site PF3) at the Antarctic Polar Front (APF), and along a nearby piston core (PS1654-2). The average length and valve area of F. kerguelensis displayed a distinct increase in the proximity of the APF, and lower values to the north and south of it, and also changed markedly over seasonal cycles, as diatom blooms and nutrient availability waxe and wane. The last glacial to interglacial transition also witnesses important changes in the general shape and valve size of $F$. kerguelensis, with specimens from glacial intervals being ca. 30\% larger than their interglacial counterparts (195 versus $150 \mu \mathrm{m}^{2}$ valve area). A sharp peak in average area and a very specific valve morphology correspond to the first signs of deglaciation and concurring SST (Sea Surface Temperature) increase and sea-ice retreat. The latter, together with melting icebergs, may have contributed dissolved iron to surface waters. The highest average sizes during glacial intervals are higher than what found anywhere in the Southern Ocean today. The newly proposed proxy (valve area and shape of $F$. kerguelensis) may thus prove useful for the reconstruction of the past position and nutrient characteristics of the APF and "opal belt", a region characterized by high production and export of biogenic silica, in a particularly sensitive and dynamic area such as the Southern Ocean.
\end{abstract}

(c) 2007 Elsevier B.V. All rights reserved.

Keywords: diatom; morphometry; Southern Ocean; nutrient; biogenic silica; export production

\section{Introduction}

The Southern Ocean is an HNLC (High Nutrients Low Chlorophyll) area, as here phytoplankton does not

\footnotetext{
* Corresponding author. Tel.: +49 4714831 1207; fax: +49 4714831 1149 .

E-mail addresses: gcortese@awi-bremerhaven.de (G. Cortese), rgersonde@awi-bremerhaven.de (R. Gersonde).

${ }^{1}$ Tel.: +49 4714831 1203; fax: +494714831 1149 .
}

seemingly completely utilize available nutrients, and high macro-nutrient levels correspond to lower than expected primary production. Three different hypotheses have been proposed to explain this phenomenon: insufficient light intensity, directly impacting photosynthetic efficiency; zooplankton grazing pressure; and micro-nutrients limitation. The latter, most commonly accepted, scenario is referred to as the "iron hypothesis" [1,2], suggesting that low levels of the micro-nutrient iron in the upper 
water column limit phytoplankton growth in some oceanic areas (e.g. the Southern Ocean, the North Pacific, the equatorial Pacific Ocean). Large-scale addition of dissolved iron to these areas results in massive phytoplankton blooms (a 30 times increase in chlorophyll levels has been the result of the fertilization experiment described in [3]), with diatoms being particularly strongly affected. This has interesting consequences for global biogeochemical cycles, as the limitation of diatom growth by low micro-nutrient levels (particularly iron) leads to higher $\mathrm{Si} / \mathrm{N}$ uptake ratios than if the conditions were ironrepleted. This induces a secondary dissolved silicon limitation of diatom growth, that sets in earlier than nitrate limitation, thus letting nitrate unused, and therefore enriched in the upper water column.

The effect of iron limitation in slowing down diatom blooms, and in changing their $\mathrm{Si} / \mathrm{N}$ uptake ratios, has been demonstrated both by culturing and incubating diatoms $[4,5]$ and by several iron fertilization experiments in the Southern Ocean [6], in the equatorial Pacific [3,7], and in the North Pacific [1]. Another very interesting aspect of iron-limited diatom blooms is connected to the observation that, during glacials, iron limitation is relieved in the Southern Ocean, and therefore the $\mathrm{Si} / \mathrm{N}$ uptake ratios of phytoplankton change dramatically between glacial and interglacial time periods. This has far reaching consequences, as the Southern Ocean acts as a major source of nitrate or dissolved silicon to low latitude coastal upwelling systems [8]. Changes in this nutrient supply, particularly switches from dominantly nitrate to dissolved silicon export, imply drastic modifications of the ecosystem structure in areas, such as coastal upwelling systems, that are responsible for most of the export of organic matter to the sediments. This, in turn, has potentially a direct impact on the functioning of the biological pump (the efficiency of organic matter export from the surface ocean) and ultimately on the sequestration of atmospheric $\mathrm{CO}_{2}$ into the ocean.

As these processes are not entirely resolved, we developed a new diatom-based tool to reconstruct nutrient cycling, ecosystem changes, and biogenic silica export in the Southern Ocean. In fact, diatoms are a major component of phytoplankton in the Southern Ocean, represent $40 \%$ of total production and make up to $75 \%$ of the total biomass in oceanic, highly productive systems [9], and are therefore prominent tools in reconstructions of past ecosystem changes and their impact on biogeochemical cycles. The initial stimulus for this work comes from a paper published $30 \mathrm{yr}$ ago [10]. In their analysis of diatom assemblages from plankton samples collected along latitudinal transect stations occupied during Leg 35 of the Deep Sea Drilling Project, these authors also studied the morphological variability of Fragilariopsis kerguelensis in ten samples, spanning 69 to $55^{\circ} \mathrm{S}$ in the Pacific Sector of the Southern Ocean, and derived an index $\left(F^{*}\right.$, defined in the Methods section and also used by us for comparison to their data) of valve shape and "compactness" for this species. This index changed markedly over the transect they examined, suggesting how the size of this diatom species might vary in response to the different oceanographic regimes in the ACC. We expanded their approach by including a total of sixty-four surface sediment samples as representative of modern-day conditions, and adding the study of sediment trap and piston core samples in order to provide timeseries of past changes in the morphology of this species. Another major difference from that study is the emergence and revamping, since then, of the abovementioned "iron hypothesis" [1,2].

Today, diatoms dominate the Southern Ocean phytoplankton, with the only exception being represented by early spring blooms south of the Antarctic Polar Front, where Phaeocystis (Haptophyta) is more abundant. Within diatoms, the most abundant and widespread species in the surface sediments is, by far, $F$. kerguelensis, an endemic Southern Ocean species whose abundance reaches up to $90 \%$ of the total diatom assemblage [11]. A possible influence of nutrients content on the morphology of $F$. kerguelensis was first mentioned in [12], who indicates the possibility of a separate variety (F. kerguelensis var. ovalis, thinner shells and smaller pores), more abundant than F. kerguelensis to the north of the Polar Front, in the Subantarctic. She related this to nutrient supply, and not to SST (as [10] later did), as F. kerguelensis is a quite eurythermal diatom species $\left(-1\right.$ to $\left.13{ }^{\circ} \mathrm{C}\right)$, and observed that high phytoplankton concentrations are found to the south of the APF, while lower phytoplankton concentrations prevail north of it. Unfortunately, however, the newly defined variety $F$. kerguelensis var. ovalis never received wider acceptance in later studies.

We therefore selected F. kerguelensis due to its abundance, its good preservation in the sediments, and the important role it has been demonstrated to play in the cycling of nutrients, and in the export of biogenic silica from the surface ocean to the sediments [13], in order to develop a new, diatom-based proxy that can help us understand the cycling of nutrients in the Southern Ocean. Changes in valve size may also provide clues over a variety of processes which are difficult to trace in the fossil record, including development of mechanically stronger frustules as protection against predators [14], ecosystem modifications linked to the presence of different grazers [15], or to different life strategies/ 
adaptations to Fe-limited conditions, also reflected in changes in abundance and dominance of diatom species, e.g. Chaetoceros spp. versus F. kerguelensis [16]. An interesting application of the proxy we developed in this work is to analyze if and how the morphology of $F$. kerguelensis varied over glacial to interglacial transitions at the Polar Front, as this may reflect millennial-scale shifts in the position of this front, and the associated high export of biogenic silica. The core we chose for this purpose is piston core PS1654-2, collected at the Polar Front in the Atlantic Sector (close to the PF3 sediment trap location), and extensively analyzed by [17], who documented its paleotemperature evolution (derived from diatom-based transfer functions), and the presence of a major meltwater event, time-correlative to the Antarctic Cold Reversal (ACR), at ca. 13 ka. The morphological variability of $F$. kerguelensis has thus been documented both in space (surface sediment samples collected over a vast portion of the areal distribution of this species in the Southern Ocean), and in time, at both a monthly/seasonal scale (sediment trap) and on millennial scale (sediment core PS1654-2). The sediment trap and piston core part of this study focuses on the Antarctic Polar Front, as this oceanographic feature represents an area of massive biogenic silica export and a major oceanographic boundary, south of which waters with high dissolved silicon are dominant [18]. At the APF, moreover, Circumpolar Deep Water and subantarctic Surface Water mix, sink, and give rise to Antarctic Intermediate Water (AAIW), which exports nutrients, nitrate in particular under modern interglacial conditions, from the Southern Ocean towards lower latitudes.

The AAIW is fundamental for the replenishment in nutrients of low latitude upwelling systems, and is of primary importance to characterize it, as its spreading path and nutrient content probably changed over glacialinterglacial cycles. Our aim will be to provide a tool to trace the oceanic and nutrient conditions south of the Polar Front, the source area for AAIW and a zone of high biogenic silica production and export (the so called "opal belt"). As today the dissolved silicon "stripping" (i.e. the conversion of dissolved silicon into biogenic silica hardparts, which are exported to the deep ocean) of the surface waters in this area is largely controlled by diatoms, we will concentrate on the main signal carrier within this group, i.e. F. kerguelensis, arguably the most important silica sinker at the APF.

\section{Material and methods}

The studied surface sediment samples were collected by a multicorer device during several RV "Polarstern" cruises to the Southern Ocean (Fig. 1, Table 1), and were selected in order to achieve maximum areal coverage, and to document a variety of oceanographic conditions in the study area. The sediment trap samples [19] were collected by a large-aperture Kiel-type sediment trap at Site PF3 $\left(50^{\circ} 08^{\prime} \mathrm{S}, 05^{\circ} 50^{\prime} \mathrm{E}\right)$, located at the Antarctic Polar Front, in the Atlantic Sector of the Southern Ocean (Fig. 1, Table 2), quite close to the position $\left(50^{\circ} 09^{\prime} \mathrm{S}, 05^{\circ} 43^{\prime} \mathrm{E}\right)$ of piston core PS1654-2 (Fig. 1, Table 3), also included in this study.

Sample treatment and preparation of slides for light microscopy documentation of diatoms followed the standard method described by [20], while the age model of core PS1654-2 is from [17], with ages converted from ${ }^{14} \mathrm{C}$-radiocarbon years to calendar years, and the application of an 800 year reservoir correction.

Pictures of an adequate number of $F$. kerguelensis specimens (an average of 38 specimens per sample) have been taken with a videocamera attached to a Zeiss Axioskop microscope, at $1000 \times$ magnification. The public domain ImageJ software (freely available from the National Institute of Health Webpage, http://rsb.info.nih. gov/ij) has been used to measure length, width, length of 5 costae for each of these specimens. A derived variable $\left(F^{*}\right.$, defined as valve length* length of 5 costae/valve width by [10]) has also been calculated, in order to directly compare our results to their findings. This variable was used by these authors as a "general shape and costae density" index and can thus give indications on the robustness and compactness of $F$. kerguelensis valves as, all other things being equal, lower $F^{*}$ values correspond to denser costae and therefore more mechanically resistant valves (i.e., following its definition, $F^{*}$ is a measure of costae density "weighted" by valve length and width). Moreover, the density of costae is frequently expressed, in diatom taxonomic descriptions, as number of costae per $10 \mu \mathrm{m}$, and the length of five costae measurement can easily be converted to such a system. The species $F$. kerguelensis is a junior synonym of Nitzschia kerguelensis [21] and, according to the definition of $N$. kerguelensis (O'Meara) Hasle reported in [10], it is characterized by $5-8$ transapical costae per $10 \mu \mathrm{m}$, which perfectly matches our estimates (5.7 to 7.8 transapical costae per $10 \mu \mathrm{m}$ in coretops).

We interpolated temperature, salinity, dissolved silicon and nitrate data for the relevant month/season at the sixtyfour surface sediment sample sites by using the multiplatform oceanography visualization software Ocean Data View [22]. This procedure was carried out automatically based on the geographic position of our cores and a regular overlay grid represented by the one degreespaced World Ocean Atlas 2001 data [23]. The geographic distribution of SST, dissolved silicon and nitrate (annual averages) in the study area is shown in Fig. 1. 

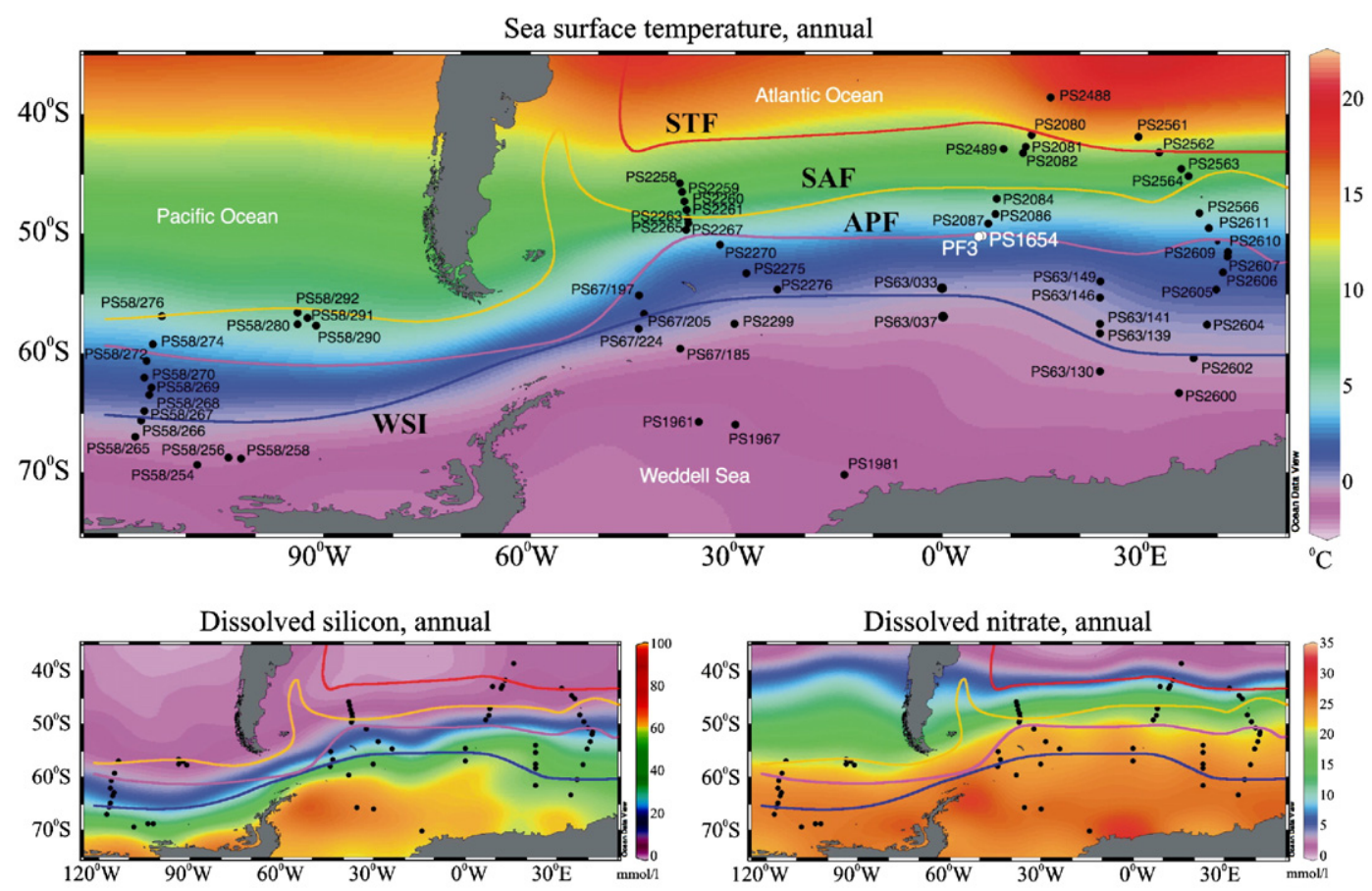

Fig. 1. Sample location, SST and nutrient distribution. Location of surface sediment samples (black dots), sediment trap $\left(\mathrm{PF} 3,50^{\circ} 08^{\prime} \mathrm{S}, 05^{\circ} 50^{\prime} \mathrm{E}\right)$ and piston core (PS1654-2, 50 $09^{\prime} .45 \mathrm{~S}, 05^{\circ} 43^{\prime} .35 \mathrm{E}$ ) locations analyzed in this study, and oceanic frontal positions and maximum sea ice extent according to [30]: Subtropical Front (STF, red), Subantarctic Front (SAF, yellow), Polar Front (APF, purple), winter sea ice edge (WSI, blue). Annually averaged values for SST, dissolved silicon and nitrate at $10 \mathrm{~m}$ water depth are also shown. Raw data from the World Ocean Atlas 2001 [23] have been gridded with the OceanDataView software [22].

All data discussed in this paper are shown in Tables $1-3$.

The programs Le Progiciel $R$ and $R d a / C c a[24]$ were used to perform Canonical Redundancy Analysis (RDA) and Principal Component Analysis (PCA) and characterize our stations in terms of environmental (RDA) and diatoms size (PCA) variables. The details of these analyses, their interpretation, and cross-correlation tables between morphometric and environmental variables are shown in the Appendix.

\section{Results}

\subsection{Surface sediments}

The average length of $F$. kerguelensis valves in the surface sediment samples (Figs. 2A and 3, Table 1) varies, over the study area, from a minimum of 25 to a maximum of $41 \mu \mathrm{m}$. The geographic distribution of this variable displays a broad band of higher than average values (ca. 35 to $40 \mu \mathrm{m}$ ) between 45 and $60^{\circ} \mathrm{S}$ in the Indian Sector, with lower values both north and south of it. This band, centered around $55^{\circ} \mathrm{S}$, and the general pattern with smaller sizes north and south of it, continue towards the west in the Atlantic Sector, although maximum values slightly decrease to ca. 32 $36 \mu \mathrm{m}$ in the Argentine Basin. In the Pacific Sector, values similar to these are found between 60 and $67^{\circ} \mathrm{S}$, again with lower values to the north and south of this band. Particularly low values are found along the $90^{\circ} \mathrm{W}$ meridian in the Pacific Sector, in the central Weddell Sea $\left(30^{\circ} \mathrm{W}\right)$, south of $62^{\circ} \mathrm{S}$, north of $48^{\circ} \mathrm{S}$ and in the Cape Basin (north of $40^{\circ} \mathrm{S}$ ) in the Atlantic-Indian Sector. A practically identical pattern can be seen in the average width measurements (Fig. 2B), which have a smaller range of variation (from 6 to $9 \mu \mathrm{m}$ ), with the only difference from the length distribution being slightly different than expected width values in the western South Atlantic (higher), and in the Weddell Sea (lower).

As a consequence of this relatively good correspondence between length and width, the average area distribution follows the pattern already described for the valve length, and the pattern is, if anything, even more clearly identifiable. Average values range from 84 to $190 \mu \mathrm{m}^{2}$, with minimum values in the Weddell Sea and Cape Basin, and maximum values around $50-55^{\circ} \mathrm{S}$ in the Atlantic-Indian Sector, particularly in the eastern part (Fig. 2C). 
Table 1

Surface sediment samples

\begin{tabular}{|c|c|c|c|c|c|c|c|c|c|c|c|c|c|c|}
\hline \multicolumn{15}{|l|}{ Coretops } \\
\hline Sample & Latitude & Longitude & Basin & Specimens & $\begin{array}{l}\text { Average } \\
\text { length }\end{array}$ & $\begin{array}{l}\text { Average } \\
\text { width }\end{array}$ & $\begin{array}{l}\text { Average } \\
5 \text { costae }\end{array}$ & $\begin{array}{l}\text { Average } \\
F^{*}\end{array}$ & $\begin{array}{l}\text { Average } \\
\text { area }\end{array}$ & $\begin{array}{l}\text { SD } \\
\text { length }\end{array}$ & $\begin{array}{l}\text { SD } \\
\text { width }\end{array}$ & $\begin{array}{l}\text { SD } 5 \\
\text { costae }\end{array}$ & $\begin{array}{l}\text { SD } \\
F^{*}\end{array}$ & $\begin{array}{l}\mathrm{SD} \\
\text { area }\end{array}$ \\
\hline PS2561-1 & -41.860 & 28.547 & Indian & 38 & 30.90 & 7.97 & 8.07 & 31.78 & 127.65 & 10.38 & 1.53 & 1.22 & 11.77 & 58.81 \\
\hline PS2562-1 & -43.183 & 31.577 & Indian & 37 & 29.44 & 7.52 & 8.01 & 31.69 & 117.27 & 10.63 & 1.82 & 1.02 & 10.64 & 66.37 \\
\hline PS2563-3 & -44.563 & 34.793 & Indian & 37 & 31.09 & 7.40 & 6.75 & 28.32 & 118.46 & 11.15 & 1.01 & 0.68 & 9.65 & 51.77 \\
\hline PS2564-2 & -45.133 & 35.902 & Indian & 37 & 33.96 & 7.63 & 7.25 & 32.39 & 135.90 & 16.26 & 1.29 & 1.04 & 16.11 & 83.67 \\
\hline PS2566-1 & -48.257 & 37.475 & Indian & 37 & 38.40 & 8.62 & 8.36 & 37.48 & 173.10 & 12.48 & 1.87 & 1.09 & 12.02 & 79.13 \\
\hline PS2611-3 & -49.505 & 38.827 & Indian & 37 & 32.89 & 8.09 & 8.24 & 33.79 & 140.47 & 11.56 & 1.68 & 1.12 & 11.69 & 76.22 \\
\hline PS2610-2 & -50.678 & 40.122 & Indian & 37 & 39.51 & 8.72 & 8.16 & 36.91 & 179.53 & 16.44 & 1.47 & 0.84 & 14.07 & 96.35 \\
\hline PS2609-2 & -51.497 & 41.598 & Indian & 61 & 40.23 & 9.07 & 8.70 & 38.68 & 189.86 & 14.86 & 1.61 & 1.05 & 13.40 & 92.97 \\
\hline PS2607-1 & -51.890 & 41.522 & Indian & 38 & 33.31 & 7.79 & 8.38 & 35.38 & 140.62 & 13.80 & 1.97 & 1.07 & 10.01 & 90.94 \\
\hline PS2606-3 & -53.220 & 40.868 & Indian & 42 & 40.85 & 8.73 & 8.63 & 40.70 & 183.74 & 14.65 & 1.46 & 0.97 & 15.41 & 82.21 \\
\hline PS63/149-2 & -53.954 & 23.048 & Indian & 39 & 32.81 & 7.47 & 8.48 & 37.96 & 126.67 & 8.42 & 1.67 & 0.71 & 9.51 & 52.35 \\
\hline PS2605-1 & -54.657 & 39.922 & Indian & 44 & 34.07 & 8.03 & 8.33 & 35.28 & 143.81 & 12.22 & 1.64 & 0.99 & 11.23 & 70.43 \\
\hline PS63/146-1 & -55.321 & 22.999 & Indian & 41 & 29.84 & 6.87 & 8.41 & 36.85 & 104.91 & 7.59 & 1.09 & 0.62 & 9.26 & 37.95 \\
\hline PS63/141-2 & -57.515 & 22.984 & Indian & 37 & 32.26 & 7.56 & 8.36 & 35.87 & 125.87 & 9.95 & 1.39 & 0.45 & 9.44 & 58.87 \\
\hline PS2604-4 & -57.598 & 38.590 & Indian & 41 & 37.80 & 7.87 & 8.62 & 41.95 & 154.72 & 12.45 & 1.72 & 0.81 & 13.36 & 71.45 \\
\hline PS63/139-2 & -58.299 & 23.001 & Indian & 36 & 29.98 & 7.31 & 8.23 & 34.07 & 113.06 & 8.08 & 1.42 & 0.68 & 8.29 & 49.39 \\
\hline PS2602-3 & -60.375 & 36.582 & Indian & 44 & 35.52 & 7.60 & 8.72 & 40.72 & 142.80 & 12.70 & 1.70 & 0.73 & 11.29 & 78.61 \\
\hline PS63/130-2 & -61.500 & 23.001 & Indian & 37 & 29.11 & 7.63 & 8.53 & 32.66 & 114.41 & 6.22 & 1.33 & 0.75 & 5.77 & 41.31 \\
\hline PS2600-1 & -63.312 & 34.507 & Indian & 32 & 28.77 & 7.18 & 8.15 & 32.94 & 108.06 & 7.35 & 1.61 & 0.90 & 7.54 & 49.82 \\
\hline PS58/292-1 & -56.567 & -93.794 & Pacific & 32 & 32.65 & 7.57 & 7.19 & 31.51 & 129.02 & 13.21 & 1.75 & 0.88 & 12.61 & 67.99 \\
\hline PS58/276-1 & -56.890 & -113.573 & Pacific & 38 & 30.57 & 7.33 & & & 114.44 & 9.44 & 1.13 & 0.82 & 10.80 & 45.30 \\
\hline PS58/291-3 & -57.037 & -92.380 & Pacific & 39 & 31.78 & 7.86 & 7.14 & 29.09 & 131.09 & 9.69 & 1.84 & 0.85 & 7.89 & 62.95 \\
\hline PS58/280-1 & -57.545 & -93.828 & Pacific & 36 & 29.45 & 7.47 & 7.25 & 28.63 & 114.38 & 9.40 & 1.37 & 0.79 & 8.79 & 53.30 \\
\hline PS58/290-1 & -57.647 & -91.155 & Pacific & 36 & 25.30 & 6.88 & 6.87 & 25.07 & 91.64 & 8.67 & 1.39 & 0.77 & 6.71 & 48.08 \\
\hline PS58/274-4 & -59.207 & -114.888 & Pacific & 36 & 32.61 & 8.10 & 8.26 & 33.60 & 135.78 & 10.69 & 1.45 & 0.95 & 11.61 & 58.35 \\
\hline PS58/272-4 & -60.610 & -115.837 & Pacific & 43 & 31.92 & 8.04 & 7.92 & 31.39 & 134.11 & 11.18 & 1.51 & 0.89 & 9.74 & 68.88 \\
\hline PS58/270-1 & -62.028 & -116.123 & Pacific & 39 & 34.06 & 8.42 & 8.40 & 33.88 & 151.14 & 12.54 & 1.71 & 0.92 & 10.37 & 82.83 \\
\hline PS58/269-4 & -62.853 & -115.077 & Pacific & 43 & 31.90 & 8.16 & 8.23 & 32.27 & 135.92 & 10.81 & 1.56 & 0.99 & 10.14 & 67.78 \\
\hline PS58/268-1 & -63.458 & -115.390 & Pacific & 52 & 31.35 & 7.82 & 8.21 & 32.81 & 128.47 & 10.14 & 1.60 & 0.88 & 8.68 & 62.36 \\
\hline PS58/267-4 & -64.827 & -116.157 & Pacific & 45 & 33.24 & 8.22 & 8.34 & 33.87 & 142.46 & 12.14 & 1.40 & 1.00 & 12.25 & 70.55 \\
\hline PS58/266-4 & -65.623 & -116.625 & Pacific & 39 & 35.41 & 8.22 & 8.49 & 36.18 & 152.56 & 12.21 & 1.49 & 0.87 & 9.70 & 75.46 \\
\hline PS58/265-1 & -66.978 & -117.443 & Pacific & 40 & 33.20 & 7.72 & 8.28 & 35.74 & 133.96 & 13.47 & 1.44 & 0.99 & 13.10 & 80.55 \\
\hline PS58/256-1 & -68.712 & -103.897 & Pacific & 39 & 31.66 & 7.03 & 8.10 & 36.38 & 118.80 & 13.61 & 1.40 & 1.22 & 14.19 & 80.66 \\
\hline PS58/258-1 & -68.767 & -102.088 & Pacific & 37 & 28.01 & 7.11 & 8.08 & 32.41 & 101.75 & 7.53 & 1.24 & 0.83 & 9.74 & 39.49 \\
\hline PS58/254-2 & -69.313 & -108.450 & Pacific & 32 & 29.74 & 6.95 & 8.04 & 34.59 & 108.53 & 11.01 & 1.48 & 0.75 & 11.56 & 63.36 \\
\hline PS2488-1 & -38.557 & 15.802 & Atlantic & 32 & 28.25 & 7.79 & 8.02 & 29.41 & 114.79 & 7.03 & 1.81 & 1.01 & 6.24 & 55.30 \\
\hline PS2080-1 & -41.717 & 13.047 & Atlantic & 33 & 26.51 & 7.21 & 6.67 & 24.39 & 102.40 & 11.45 & 1.87 & 1.15 & 9.21 & 62.61 \\
\hline PS2081-1 & -42.693 & 12.192 & Atlantic & 41 & 30.44 & 7.19 & 6.87 & 28.95 & 114.31 & 15.21 & 1.43 & 0.92 & 13.68 & 67.24 \\
\hline PS2489-4 & -42.883 & 8.968 & Atlantic & 39 & 25.80 & 6.35 & 6.40 & 26.11 & 84.38 & 9.64 & 1.06 & 0.65 & 9.60 & 40.87 \\
\hline PS2082-3 & -43.218 & 11.759 & Atlantic & 40 & 28.94 & 7.00 & 6.66 & 27.54 & 105.81 & 10.98 & 1.36 & 0.73 & 9.55 & 53.22 \\
\hline PS2491-3 & -44.955 & 5.970 & Atlantic & 38 & 32.72 & 8.00 & 7.33 & 30.47 & 132.70 & 11.66 & 1.44 & 0.94 & 11.74 & 55.29 \\
\hline PS2258-1 & -45.755 & -38.173 & Atlantic & 36 & 32.19 & 8.01 & 7.50 & 30.51 & 135.12 & 12.56 & 1.71 & 1.22 & 12.64 & 72.03 \\
\hline PS2259-1 & -46.485 & -37.851 & Atlantic & 33 & 31.61 & 8.10 & 7.53 & 30.00 & 133.10 & 12.84 & 1.68 & 1.06 & 14.03 & 70.67 \\
\hline PS2084-2 & -47.025 & 7.959 & Atlantic & 35 & 28.25 & 7.29 & 7.04 & 27.32 & 105.31 & 11.58 & 0.91 & 0.63 & 10.78 & 50.47 \\
\hline PS2260-1 & -47.267 & -37.507 & Atlantic & 37 & 31.61 & 8.13 & 7.55 & 29.22 & 140.68 & 13.20 & 2.29 & 1.12 & 9.50 & 88.88 \\
\hline PS2261-1 & -47.992 & -37.191 & Atlantic & 35 & 29.73 & 8.15 & 7.47 & 27.12 & 129.57 & 12.74 & 1.79 & 1.14 & 10.83 & 76.31 \\
\hline PS2086-3 & -48.319 & 7.757 & Atlantic & 42 & 24.90 & 7.41 & 7.05 & 23.96 & 94.13 & 6.48 & 1.06 & 0.78 & 7.15 & 34.63 \\
\hline PS2263-1 & -48.723 & -36.990 & Atlantic & 32 & 30.31 & 8.17 & 7.73 & 29.27 & 128.15 & 10.29 & 1.55 & 1.03 & 11.39 & 59.22 \\
\hline PS2265-2 & -49.066 & -36.942 & Atlantic & 51 & 36.25 & 8.74 & 7.72 & 31.96 & 166.52 & 13.34 & 1.70 & 0.98 & 10.55 & 88.26 \\
\hline PS2087-1 & -49.134 & 6.706 & Atlantic & 46 & 35.27 & 8.21 & 7.57 & 32.69 & 152.59 & 13.44 & 1.65 & 1.05 & 12.29 & 81.34 \\
\hline PS2267-2 & -49.632 & -37.272 & Atlantic & 35 & 34.57 & 8.72 & 8.03 & 32.07 & 157.98 & 11.96 & 1.75 & 0.99 & 10.42 & 77.84 \\
\hline PS2270-5 & -50.882 & -32.321 & Atlantic & 41 & 31.55 & 8.80 & 7.46 & 26.62 & 144.34 & 8.62 & 1.53 & 0.90 & 6.16 & 60.85 \\
\hline PS2275-1 & -53.284 & -28.539 & Atlantic & 37 & 32.26 & 8.20 & 7.70 & 30.52 & 137.80 & 11.94 & 1.54 & 1.17 & 11.57 & 68.38 \\
\hline PS63/033-5 & -54.521 & -0.007 & Atlantic & 35 & 39.12 & 8.64 & 8.50 & 37.84 & 180.28 & 14.87 & 1.91 & 1.02 & 10.76 & 97.98 \\
\hline PS2276-1 & -54.631 & -23.970 & Atlantic & 51 & 34.25 & 8.48 & 7.87 & 31.39 & 152.61 & 12.88 & 1.60 & 0.82 & 9.26 & 79.60 \\
\hline PS67/197-4 & -55.143 & -44.094 & Atlantic & 39 & 31.13 & 7.91 & 7.47 & 29.94 & 128.40 & 12.95 & 1.41 & 1.12 & 14.79 & 71.40 \\
\hline
\end{tabular}


Table 1 (continued)

\begin{tabular}{|c|c|c|c|c|c|c|c|c|c|c|c|c|c|c|}
\hline \multicolumn{15}{|l|}{ Coretops } \\
\hline Sample & \multirow{2}{*}{$\begin{array}{rr}\text { Latitude } \\
-4-56.701\end{array}$} & \multirow{2}{*}{$\begin{array}{l}\text { Longitude } \\
-43.361\end{array}$} & \multirow{2}{*}{ Atlantic } & \multirow{2}{*}{39} & \multirow{2}{*}{$\begin{array}{l}\text { length } \\
28.83\end{array}$} & \multirow{2}{*}{$\begin{array}{l}\text { width } \\
8.16\end{array}$} & \multirow{2}{*}{$\begin{array}{l}5 \text { costae } \\
7.73\end{array}$} & \multirow{2}{*}{$\begin{array}{l}F^{*} \\
27.47\end{array}$} & \multirow{2}{*}{$\begin{array}{l}\text { area } \\
120.94\end{array}$} & \multirow{2}{*}{$\begin{array}{r}\text { length } \\
8.80\end{array}$} & \multirow{2}{*}{$\begin{array}{l}\text { width } \\
1.32\end{array}$} & \multirow{2}{*}{$\frac{\text { costae }}{0.80}$} & \multirow{2}{*}{$\frac{F^{*}}{8.02}$} & \multirow{2}{*}{$\frac{\text { area }}{52.34}$} \\
\hline PS67/205-4 & & & & & & & & & & & & & & \\
\hline PS63/037-4 & -56.927 & 0.099 & Atlantic & 35 & 32.10 & 7.20 & 8.21 & 36.40 & 121.06 & 12.43 & 1.25 & 0.66 & 11.36 & 68.65 \\
\hline PS2299-1 & -57.509 & -30.235 & Atlantic & 37 & 32.49 & 8.66 & 7.83 & 29.44 & 146.97 & 11.97 & 1.70 & 0.65 & 9.76 & 74.63 \\
\hline PS67/224-3 & -57.944 & -44.196 & Atlantic & 36 & 33.47 & 8.42 & 7.60 & 29.79 & 152.83 & 16.22 & 2.00 & 0.92 & 11.38 & 106.33 \\
\hline PS67/185-1 & -59.585 & -38.097 & Atlantic & 33 & 32.00 & 8.74 & 7.85 & 28.53 & 147.64 & 12.13 & 1.70 & 0.90 & 8.95 & 79.64 \\
\hline PS1961-1 & -65.720 & -35.448 & Atlantic & 31 & 32.05 & 6.99 & 8.42 & 38.81 & 115.14 & 11.52 & 0.92 & 0.82 & 14.36 & 54.22 \\
\hline PS1967-1 & -65.957 & -30.071 & Atlantic & 30 & 26.77 & 6.81 & 7.94 & 31.21 & 93.89 & 8.12 & 1.01 & 0.77 & 8.80 & 39.53 \\
\hline PS1981-1 & -70.132 & -14.254 & Atlantic & 37 & 31.06 & 6.72 & 8.28 & 38.38 & 108.07 & 11.11 & 1.10 & 0.73 & 13.19 & 52.06 \\
\hline
\end{tabular}

The following items are reported for each of the samples: geographic position and Southern Ocean Sector; number of measured specimens; average length, width, 5 costae length, $F^{*}$, and average area of the measured $F$. kerguelensis specimens, along with the corresponding standard deviations.

The average length of five costae varies from 6.4 to $8.7 \mu \mathrm{m}$ (corresponding to 7.8 and 5.7 costae per $10 \mu \mathrm{m}$, respectively). Values higher than ca. $8 \mu \mathrm{m}$ are found in the southern part of the study area (Fig. 2D, south of $47^{\circ} \mathrm{S}$ in the Indian-Atlantic Sector, south of $53^{\circ} \mathrm{S}$ in the Atlantic Sector, and south of $57^{\circ} \mathrm{S}$ in the Pacific Sector), and decrease towards the north. As a consequence of the sharp gradient shown by this variable, and of the above mentioned generally good match between length and width, the average $F^{*}$ distribution closely resembles the one for valve length and for the five costae length. Probably the only remarkable difference occurs in the Weddell Sea, where average $F^{*}$ values are higher than expected, a reflection of the occurrence there of specimens having anomalously narrow valves with longer five costae distances. Average $F^{*}$ values range from 24 to $42 \mu \mathrm{m}$ over the study area (Fig. 2E).

Due to the strong intercorrelation within the measured variables (valve area is a linear function of width and length, $F^{*}$ includes the length of five costae, width and length in its definition), we will, in the following, mostly focus on the average area of the valve, and discuss $F^{*}$ and length of five costae where relevant. The maximum area values are generally found, through the different Sectors of the Southern Ocean, in a band between the winter sea-ice edge and the Antarctic Polar Front (Fig. 2C), with a slight expansion into the Subantarctic Province in the Indian Sector. This band, with continuous supply of micro- and macronutrients by upwelling, is characterized by a specific nutrient pattern, with enough dissolved nitrate available in surface waters, and an active dissolved silicon depletion, most likely linked to an increased biogenic silica export by F. kerguelensis. The patch of high area values in the subantarctic Indian Ocean also probably relates to a very specific ecosystem, with a subsurface productivity maximum that goes undetected by using satellite chlorophyll sensors (see Appendix for a detailed comparison of our data to chlorophyll concentrations), but that has been reconstructed by inverse modelling of Particulate Organic Carbon (POC) export in this area (Fig. 8 in [25]), along with higher Th-corrected export fluxes of biogenic silica to the sediment [26]. The areas where higher POC export is not matched by particularly high opal export could represent zones where the diatom species responsible for most of the production of biogenic silica is Chaetoceros spp. (easily remineralized in the upper water column) instead of F. kerguelensis. Another interesting size pattern is visible around the Scotia Sea arc (roughly between $20-50^{\circ} \mathrm{W}$ and $50-60^{\circ} \mathrm{S}$ ), with slightly higher values outlining this topographic feature. This could indicate a positive effect of local higher $\mathrm{Fe}$ concentration in surface waters, advected from the shallower areas to the west, giving rise to larger valves.

\subsection{Sediment trap}

The average valve area of $F$. kerguelensis varies along a very marked seasonal cycle (Fig. 4, Table 2), with generally larger valves corresponding to the austral spring/early summer, and smaller valves during late summer, autumn and winter. This general size variability pattern is well reflected in the total diatom flux measurements (Fig. 4g), showing a dichotomy between autumn/winter (muted signal, practically no measurable flux at the Polar Front trap site) and much higher and variable flux values during spring and summer.

Relative abundances of F. kerguelensis (Fig. 4h, Table 2) also seem to have a strong seasonal cycle, as they fluctuate between low values during spring and summer (seasonal average $=39 \%$, minimum $=28 \%$ ) and higher values during autumn/winter (seasonal average $=63 \%$, minimum $=57 \%$ ). As $F$. kerguelensis is a particularly dissolution-resistant species, it is not clear whether the higher autumn/winter relative abundances of this species are a primary signal, or an indirect 
Table 2

Sediment trap samples

\begin{tabular}{|c|c|c|c|c|c|c|c|c|c|c|c|c|c|c|c|c|c|c|}
\hline \multicolumn{19}{|l|}{ Sediment trap } \\
\hline Sample & Latitude & Longitude & Basin & \# & $\begin{array}{l}\text { Average } \\
\text { length }\end{array}$ & $\begin{array}{l}\text { Average } \\
\text { width }\end{array}$ & $\begin{array}{l}\text { Average } \\
5 \text { costae } \\
\end{array}$ & \multicolumn{2}{|c|}{ Average $F^{*}$} & $\begin{array}{l}\text { Average } \\
\text { area }\end{array}$ & $\begin{array}{l}\text { SD } \\
\text { length }\end{array}$ & $\begin{array}{l}\text { SD } \\
\text { width }\end{array}$ & $\begin{array}{l}\text { SD } 5 \\
\text { costae }\end{array}$ & $\begin{array}{l}\mathrm{SD} \\
F^{*}\end{array}$ & $\begin{array}{l}\mathrm{SD} \\
\text { area }\end{array}$ & F.kerg.\% & & Averages \\
\hline PF3Upper1 & -50.127 & 5.833 & Atlantic & 35 & 31.18 & 7.93 & 7.40 & 29.15 & & 126.14 & 9.59 & 1.03 & 0.80 & 8.47 & 49.14 & 42.9 & Spr/Sum & F.kerg. $\%$ \\
\hline PF3Upper2 & -50.127 & 5.833 & Atlantic & 41 & 34.73 & 8.41 & 7.53 & 31.17 & & 148.38 & 10.55 & 1.01 & 0.88 & 9.23 & 55.43 & 47.8 & Spr/Sum & Spr/Sum \\
\hline PF3Upper3 & -50.127 & 5.833 & Atlantic & 39 & 31.95 & 7.93 & 7.30 & 29.57 & & 131.68 & 12.61 & 1.24 & 0.92 & 11.84 & 67.51 & 31.8 & Spr/Sum & 39.2 \\
\hline PF3Upper4 & -50.127 & 5.833 & Atlantic & 34 & 25.27 & 7.38 & 6.81 & 23.51 & & 96.50 & 8.20 & 1.21 & 0.83 & 7.33 & 45.97 & 37.6 & $\mathrm{Spr} / \mathrm{Sum}$ & \\
\hline PF3Upper5 & -50.127 & 5.833 & Atlantic & 44 & 24.07 & 7.15 & 6.65 & 22.42 & & 90.26 & 7.69 & 1.44 & 0.94 & 6.36 & 45.07 & 37.5 & Spr/Sum & Aut/Win \\
\hline PF3Upper6 & -50.127 & 5.833 & Atlantic & 39 & 26.49 & 7.06 & 6.90 & 25.90 & & 96.55 & 8.82 & 1.00 & 0.80 & 8.06 & 45.37 & 42.3 & Spr/Sum & 63.4 \\
\hline PF3Upper7 & -50.127 & 5.833 & Atlantic & 35 & 28.43 & 7.82 & 7.01 & 25.66 & & 116.91 & 10.00 & 1.64 & 0.80 & 9.28 & 62.26 & 58.1 & Aut/Win & \\
\hline PF3Upper8 & -50.127 & 5.833 & Atlantic & 37 & 25.34 & 7.29 & 6.62 & 23.16 & & 94.43 & 6.72 & 1.17 & 0.67 & 6.17 & 35.43 & 64.5 & Aut/Win & Area \\
\hline PF3Upper9 & -50.127 & 5.833 & Atlantic & 35 & 25.10 & 6.94 & 6.67 & 23.94 & & 91.64 & 10.66 & 1.16 & 0.72 & 9.32 & 52.28 & 65.7 & Aut/Win & Spr/Sum \\
\hline PF3Upper10 & -50.127 & 5.833 & Atlantic & 40 & 33.98 & 7.72 & 7.35 & 32.27 & & 139.13 & 14.82 & 1.56 & 0.94 & 14.22 & 81.78 & 57.3 & Aut/Win & 123.06 \\
\hline PF3Upper11 & -50.127 & 5.833 & Atlantic & 50 & 27.48 & 7.03 & 6.90 & 26.82 & & 102.25 & 10.67 & 1.42 & 0.80 & 9.27 & 61.47 & 66.2 & Aut/Win & \\
\hline PF3Upper12 & -50.127 & 5.833 & Atlantic & 38 & 26.26 & 6.81 & 7.29 & 28.24 & & 93.51 & 11.00 & 1.27 & 0.87 & 11.30 & 53.40 & 68.7 & Aut/Win & Aut/Win \\
\hline PF3Upper13 & -50.127 & 5.833 & Atlantic & 33 & 34.03 & 7.17 & 7.74 & 36.50 & & 126.23 & 14.78 & 0.98 & 0.97 & 14.99 & 67.72 & 49.6 & Spr/Sum & 106.31 \\
\hline PF3Upper14 & -50.127 & 5.833 & Atlantic & 38 & 38.32 & 7.69 & 7.50 & 37.31 & & 151.26 & 15.43 & 0.98 & 0.85 & 14.87 & 71.22 & 27.8 & Spr/Sum & \\
\hline PF3Upper15 & -50.127 & 5.833 & Atlantic & 37 & 34.17 & 7.52 & 7.57 & 34.97 & & 132.37 & 13.63 & 1.16 & 1.08 & 15.53 & 64.56 & 38.1 & Spr/Sum & \\
\hline PF3Upper16 & -50.127 & 5.833 & Atlantic & 42 & 31.24 & 7.40 & 7.12 & 29.90 & & 120.87 & 14.76 & 1.03 & 1.00 & 12.96 & 73.44 & 35.6 & Spr/Sum & \\
\hline PF3Upper17 & -50.127 & 5.833 & Atlantic & 38 & 33.12 & 7.72 & 7.18 & 30.64 & & 133.41 & 12.62 & 1.31 & 0.83 & 10.17 & 66.32 & 40.4 & Spr/Sum & \\
\hline \multicolumn{19}{|c|}{ Average Area Upper $=117.1$} \\
\hline PF3Lower1 & \multicolumn{2}{|c|}{-50.127} & 5.833 & \multicolumn{2}{|c|}{ Atlantic } & \multicolumn{2}{|c|}{36.33} & 8.48 & 1.89 & \multicolumn{2}{|c|}{33.67} & 159.88 & \multicolumn{2}{|c|}{12.82} & 1.33 & 0.9 & 10.34 & 79.35 \\
\hline PF3Lower2 & \multicolumn{2}{|c|}{-50.127} & 5.833 & Atlantic & 35 & 35 & & 8.28 & 7.37 & 31.0 & & 151.16 & 15 & & 1.25 & 0.63 & 12.22 & 85.93 \\
\hline PF3Lower3 & -50 & & & Atlantic & 39 & 34 & & 8.25 & 7.49 & 31.0 & & 144.04 & 12 & & 1.34 & 1.03 & 10.73 & 64.31 \\
\hline PF3Lower4 & -50 & & & Atlantic & 35 & 27 & & 7.63 & 7.24 & 26.5 & & 108.03 & 7.5 & & 1.12 & 0.94 & 7.87 & 42.89 \\
\hline PF3Lower5 & -50 . & & & Atlantic & 38 & 28 & & 7.81 & 7.13 & 25.7 & & 113.30 & 9.3 & & 1.05 & 0.80 & 7.78 & 47.14 \\
\hline
\end{tabular}




\begin{tabular}{|c|c|c|c|c|c|c|c|c|c|c|c|c|c|c|}
\hline PF3Lower6 & -50.127 & 5.833 & Atlantic & 37 & 30.01 & 7.87 & 6.72 & 25.71 & 121.97 & 10.03 & 1.22 & 0.92 & 8.73 & 53.39 \\
\hline PF3Lower7 & -50.127 & 5.833 & Atlantic & 38 & 24.81 & 6.78 & 6.83 & 24.91 & 87.60 & 10.11 & 1.02 & 0.66 & 9.71 & 45.33 \\
\hline PF3Lower8 & -50.127 & 5.833 & Atlantic & 34 & 28.19 & 7.54 & 7.20 & 26.98 & 111.22 & 9.66 & 1.41 & 0.92 & 8.63 & 55.35 \\
\hline PF3Lower9 & -50.127 & 5.833 & Atlantic & 47 & 26.28 & 7.08 & 6.91 & 25.26 & 97.51 & 9.80 & 1.22 & 0.63 & 6.74 & 51.53 \\
\hline PF3Lower10 & -50.127 & 5.833 & Atlantic & 37 & 29.28 & 7.63 & 7.11 & 27.38 & 117.62 & 11.12 & 1.48 & 0.96 & 9.53 & 64.52 \\
\hline PF3Lower11 & -50.127 & 5.833 & Atlantic & 40 & 28.80 & 7.51 & 7.47 & 28.67 & 114.47 & 9.82 & 1.74 & 0.95 & 8.50 & 60.63 \\
\hline PF3Lower12 & -50.127 & 5.833 & Atlantic & 39 & 27.60 & 7.26 & 7.13 & 27.01 & 105.39 & 10.97 & 1.36 & 0.84 & 9.11 & 59.96 \\
\hline PF3Lower13 & -50.127 & 5.833 & Atlantic & 32 & 34.93 & 7.71 & 7.31 & 32.77 & 142.20 & 15.37 & 1.46 & 1.13 & 13.14 & 81.09 \\
\hline PF3Lower14 & -50.127 & 5.833 & Atlantic & 38 & 31.61 & 7.62 & 7.44 & 31.17 & 124.99 & 11.76 & 1.40 & 1.00 & 11.92 & 60.26 \\
\hline PF3Lower15 & -50.127 & 5.833 & Atlantic & 38 & 29.77 & 7.50 & 7.03 & 27.81 & 117.65 & 11.48 & 1.43 & 0.85 & 10.06 & 62.82 \\
\hline PF3Lower16 & -50.127 & 5.833 & Atlantic & 38 & 27.12 & 7.23 & 7.00 & 26.20 & 102.38 & 10.95 & 1.21 & 0.68 & 9.88 & 56.55 \\
\hline PF3Lower17 & -50.127 & 5.833 & Atlantic & 34 & 30.23 & 7.66 & 7.28 & 28.89 & 122.78 & 13.11 & 1.75 & 0.82 & 12.19 & 71.82 \\
\hline
\end{tabular}

Average Area Lower $=120.1$

Monthly oceanography at trap site

\begin{tabular}{lccccrrrrrrrr}
\hline at $10 \mathrm{~m}$ & January & February & March & April & May & June & July & August & September & October & November & December \\
\hline SST & 4.06 & 4.63 & 4.66 & 4.47 & 3.40 & 3.20 & 2.38 & 3.28 & 1.90 & 1.90 & 2.63 & 3.40 \\
Diss.silicon & 12.15 & 10.85 & 11.90 & 10.32 & 14.10 & 14.93 & 23.92 & 23.98 & 24.52 & 31.71 & 25.25 & 18.97 \\
Nitrate & 19.28 & 21.96 & 20.82 & 21.56 & 22.46 & 22.61 & 18.58 & 18.59 & 18.59 & 21.07 & 21.23 & 19.67
\end{tabular}

Coretop close to trap

\begin{tabular}{|c|c|c|c|c|c|c|c|c|c|c|c|c|c|c|}
\hline Sample & Latitude & Longitude & Basin & \# & $\begin{array}{l}\text { Average } \\
\text { length }\end{array}$ & $\begin{array}{l}\text { Average } \\
\text { width }\end{array}$ & $\begin{array}{l}\text { Average } 5 \\
\text { costae }\end{array}$ & $\begin{array}{l}\text { Average } \\
F^{*}\end{array}$ & $\begin{array}{l}\text { Average } \\
\text { area }\end{array}$ & $\begin{array}{l}\text { SD } \\
\text { length }\end{array}$ & $\begin{array}{l}\text { SD } \\
\text { width }\end{array}$ & $\begin{array}{l}\text { SD } 5 \\
\text { costae }\end{array}$ & $\begin{array}{l}\mathrm{SD} \\
F^{*}\end{array}$ & $\begin{array}{l}\mathrm{SD} \\
\text { area }\end{array}$ \\
\hline PS1759-1 & -50.17 & 5.755 & Atlantic & 35 & 30.73 & 7.80 & 7.71 & 30.62 & 124.60 & 9.42 & 1.40 & 1.00 & 9.35 & 54.99 \\
\hline
\end{tabular}

The average $F$. kerguelensis valve area for the upper and lower trap samples is also reported, along with $F$. kerguelensis relative abundances for each month and their seasonal averages in the upper trap, and measurements on an additional coretop sample (PS1759-1), for direct comparison to the sediment trap data. Monthly averaged values for SST, dissolved silicon, nitrate at $10 \mathrm{~m}$ water depth are also shown. Raw data from the World Ocean Atlas 2001 [23], gridded and interpolated with the OceanDataView software [22]. 
Table 3

Sediment core samples

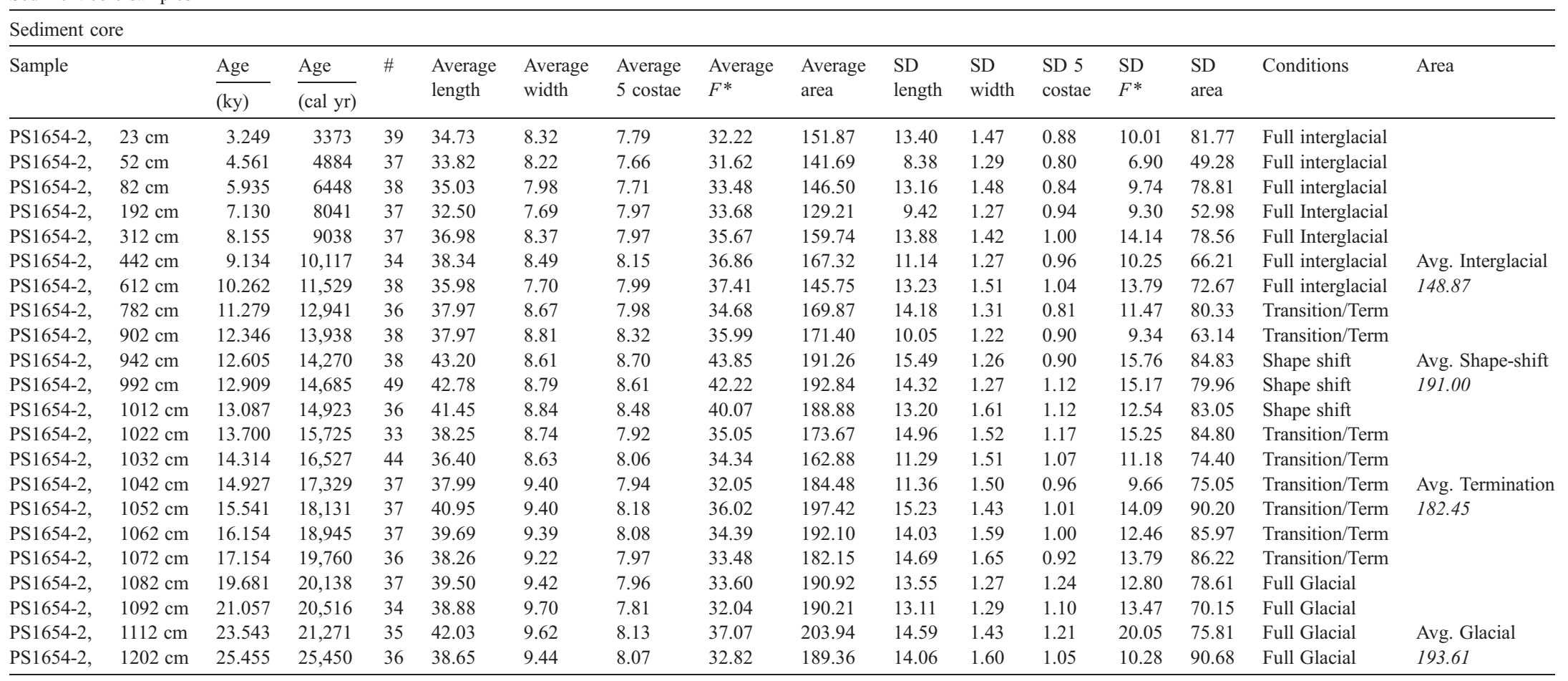

The age of each sample [17], in calendar years, with an 800 year reservoir correction, along with average area values for specific time intervals (full interglacial, termination, shape shift, full glacial), are also shown. 

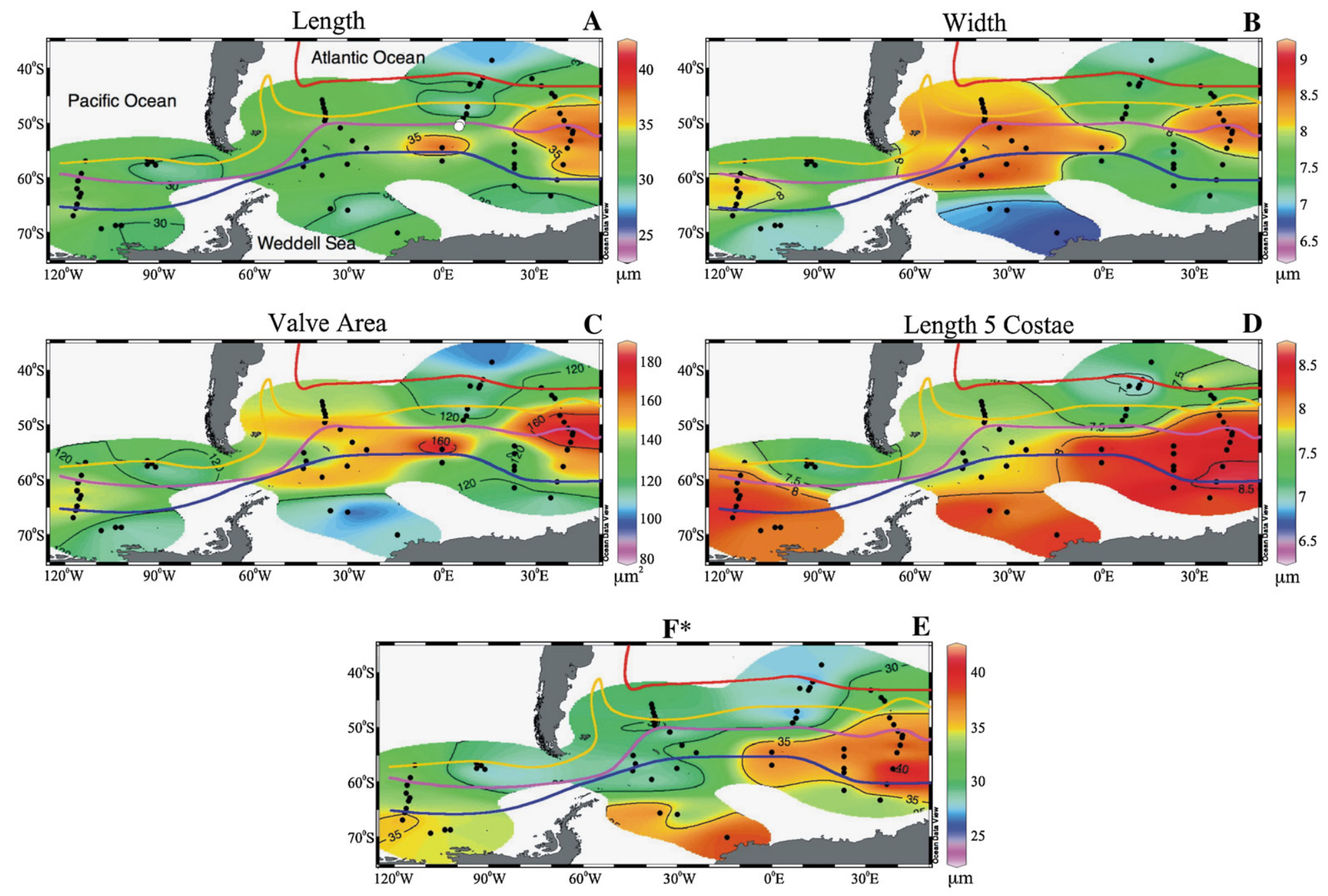

Fig. 2. A-E. Geographic distribution of morphometric variables. The color scale (in $\mu \mathrm{m}$ for all graphs but Fig. 2C, average area, in $\mu \mathrm{m}^{2}$ ) shows the geographic distribution of the following variables for F. kerguelensis: average length, width, valve area, length of five costae, and $F^{*}$ (defined in [10] as valve length* length of 5 costae/valve width). Isolines are shown to improve readability. Oceanic frontal positions (colored lines) as in Fig. 1. 


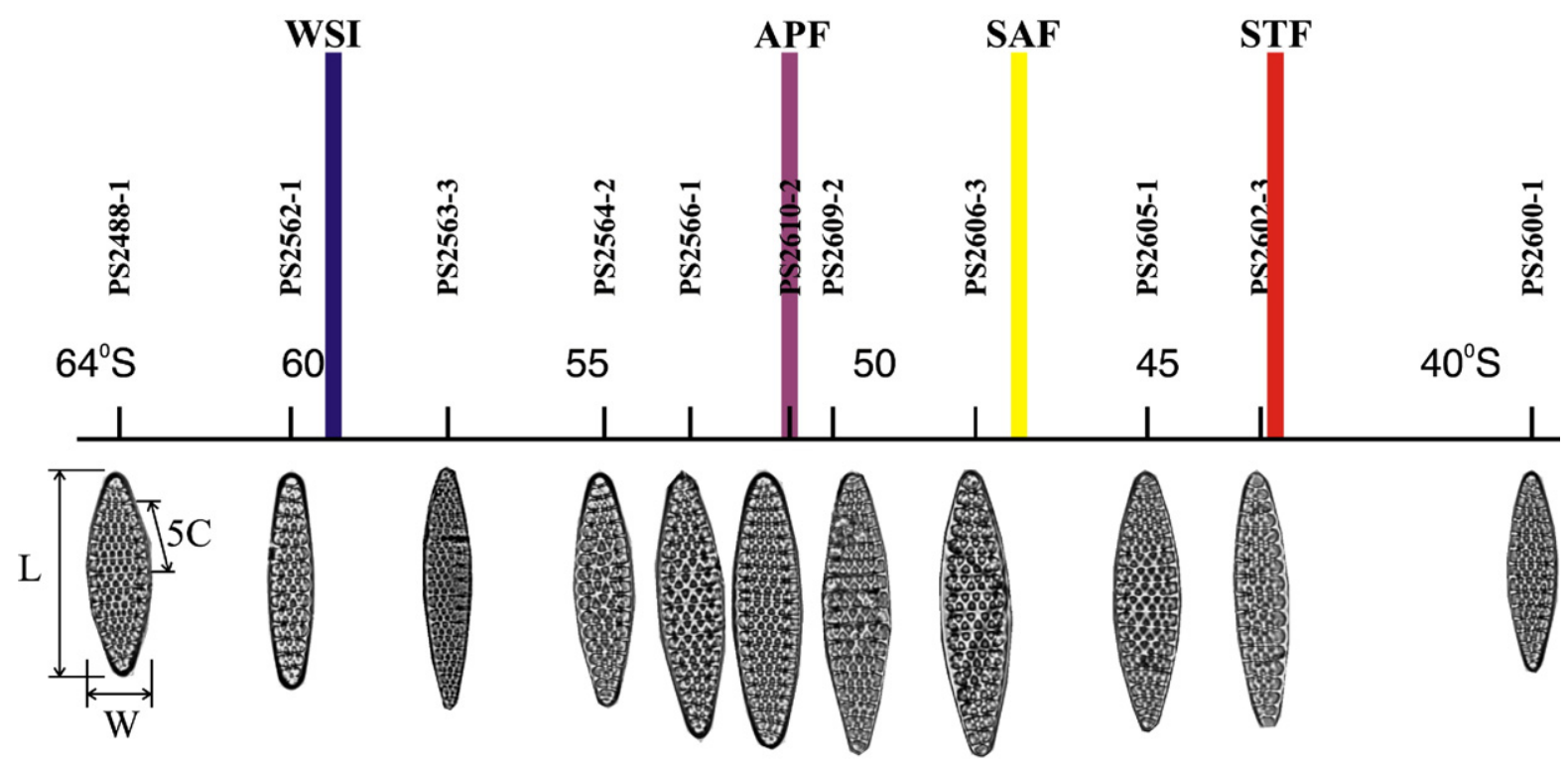

Fig. 3. Schematic transect of $F$. kerguelensis valve area. Average size of $F$. kerguelensis in surface sediments on a transect along $30^{\circ} \mathrm{E}$ longitude. Oceanic front positions (name and color coding as in Fig. 1) are also shown, along with the measured variables ( $L=$ length; $W=$ width; $5 \mathrm{C}=$ length of five costae).

enrichment due to lower diatom fluxes during these seasons, and consequent selective dissolution of the other, less dissolution resistant, species.

A detailed investigation of the sediment trap data (Fig. 4a) reveals that the average valve area is at peak values $\left(148 \mu \mathrm{m}^{2}\right.$ ) during the late spring of 1988 , it then drops rather abruptly going into the summer, and reaches minimum values during mid-February $\left(90 \mu \mathrm{m}^{2}\right)$. From mid-summer till the succeeding mid-winter (beginning of February to beginning of August), average areas do not exceed $97 \mu^{2}$, with two exceptions: sample PF3Upper7 (117 $\mu \mathrm{m}^{2}$, still quite smaller than spring equivalents) and sample PF3Upper10 $\left(139 \mu \mathrm{m}^{2}\right.$, discussed below). Starting from September 1989, valve size switches to spring mode, with an average size of $133 \mu^{2}$ for the last five samples, and a maximum value of $151 \mu \mathrm{m}^{2}$ observed at the end of October.

There is a good match between the upper and lower trap records, and exceptions to this pattern are shown by only one sample (PF3Upper10) in the upper trap timeseries, showing relatively high values during autumn and, in the deeper trap, by the relatively smaller than expected valves during the late spring of 1989 compared to those representative of the spring 1988. While the latter may be a reflection of interannual variability in bloom intensity and characteristics (and their effect on the average size of $F$. kerguelensis), we do not have a valid explanation for the larger valves in sample PF3Upper10. However, it is interesting to note how this time interval (Fig. $4 \mathrm{c}-\mathrm{h}$ ) coincides with vast reorganizations of the upper water column structure, with a decreasing trend in SST going into the winter, an increasing dissolved silicon content, and an anomalously shallow thermocline depth, all allowing a better access to the necessary macronutrients during this time interval.

\subsection{Sediment core PS1654-2}

This core covers a variety of paleoclimatic/oceanographic conditions: full interglacial (down to $10 \mathrm{ka}$ ), transition/termination (between 10 and $20 \mathrm{ka}$, including a meltwater/cooling event at ca. $13 \mathrm{ka}$ ), and full glacial (earlier than $20 \mathrm{ka}$ ). The evolution through time of the average valve area of F. kerguelensis (Figs. 5-6, Table 3) mirrors extremely well this partition, as it is at its highest (193.6 $\mu \mathrm{m}^{2}$ in average) during full glacial times, decreases to $182.4 \mu \mathrm{m}^{2}$ during the transition with a rebound towards higher, glacial values $\left(191.0 \mu \mathrm{m}^{2}\right)$ during the start of deglaciation and retreat of winter sea ice from this APF site, and settles down to $148.9 \mu^{2}$ during interglacial times.

\section{Discussion}

\subsection{Surface sediment and sediment trap PF3: F. kerguelensis under modern conditions}

The strong link between high biogenic silica export at the APF, nutrients availability and larger size in F. kerguelensis is one of the main results of our surface 


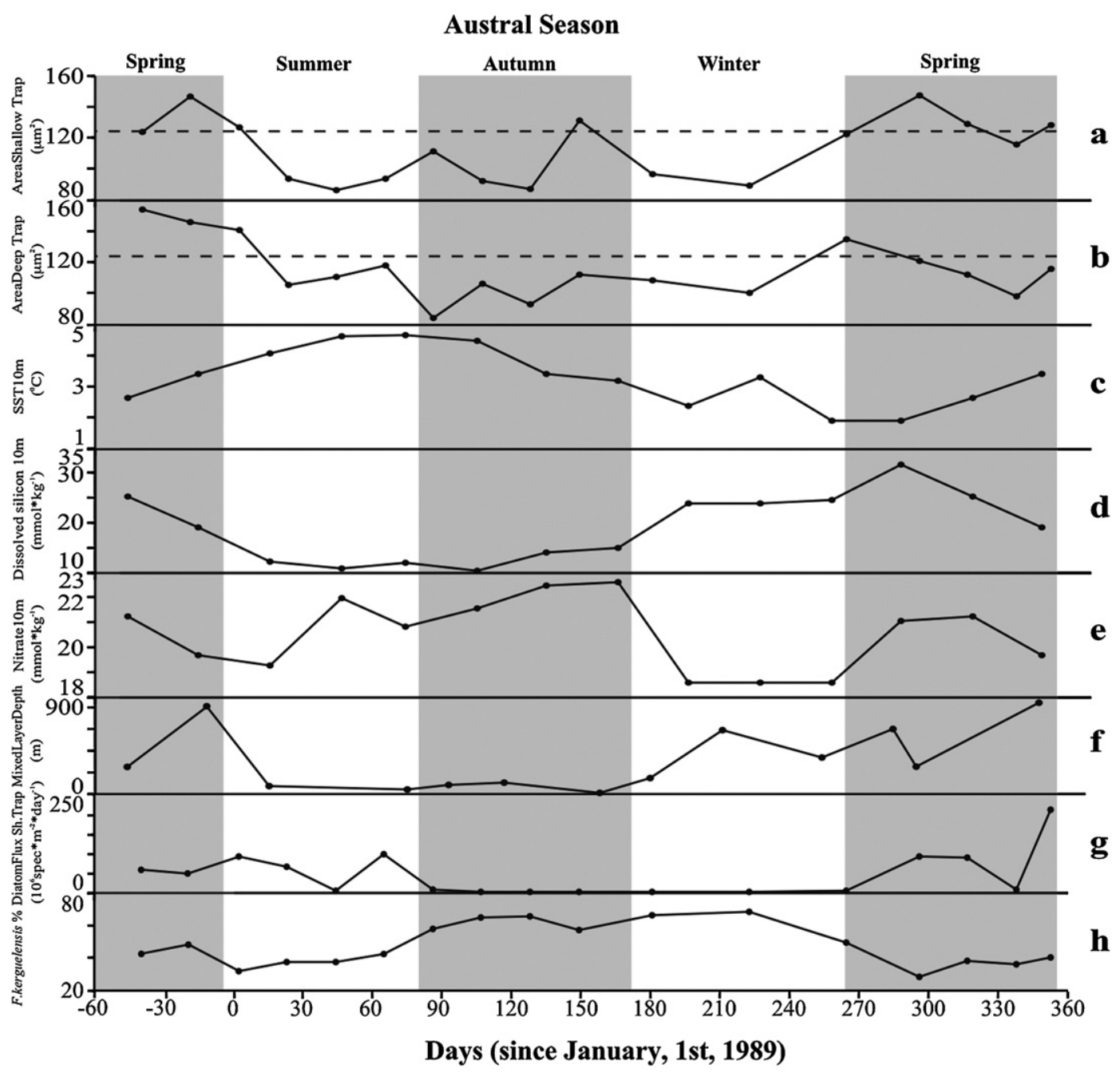

Fig. 4. a-h. Sediment trap PF3 results. Sediment trap data from the Antarctic Polar Front, in the Atlantic Sector of the Southern Ocean (Site PF3 [19]. $50^{\circ} 08^{\prime} \mathrm{S}, 05^{\circ} 50^{\prime} \mathrm{E}$ ), collected for this study (average $F$. kerguelensis area for the shallow and deep trap, panels a-b) are reported, along with SST, dissolved silicon, nitrate at $10 \mathrm{~m}$ depth, and mixed layer depth (panels c-f, all from World Ocean Atlas 2001 [23]), diatom flux (in millions of specimens $\mathrm{m}^{-2}$ day $^{-1}$ ) and F. kerguelensis relative abundance (panels $\mathrm{g}-\mathrm{h}$, both from [19]). The horizontal dashed line represents the average area of F. kerguelensis in a surface sediment sample (PS1759-1, 50 $09^{\prime} .9 \mathrm{~S}, 05^{\circ} 45^{\prime} .3 \mathrm{E}$ ) collected in the immediate proximity of the sediment trap location. The time scale is in days since January 1st, 1989, and austral hemisphere seasons are highlighted by the shading.

sediment morphometric study, as can be seen in Fig. 2C, where the average valve area, the position of the main oceanographic fronts and the winter sea-ice limit are shown. The largest $F$. kerguelensis valves are found in a band located just to the north of the winter sea-ice edge and south of the APF, where dissolved silicon is depleted very strongly by diatoms and exported as biogenic silica to the sediment (Fig. 7). The eastern part of the study area shows a similar pattern, and large valves are also found slightly to the north of this band (particularly in the Indian Sector), a feature nicely matched by independently estimated biogenic silica bottom flux [25] and Th-corrected accumulation rates
[26]. Moreover, south of the Polar Front, phytoplankton is not macronutrient limited, as the main nutrients necessary for diatom growth are present in high enough concentrations (dissolved silicon: $20-40 \mu \mathrm{mol} / 1$, nitrate: 20-25 $\mu \mathrm{mol} / \mathrm{l})$. North of the APF, the concentrations of both nutrients (particularly dissolved silicon) sharply decrease, and give rise to nutrient limitation, a possible explanation for the smaller $F$. kerguelensis valves found to the north of the APF.

We therefore propose to use high values of the average area of $F$. kerguelensis as a proxy for this zone of high biogenic silica export and high dissolved silicon depletion, where dissolved silicon is stripped by diatoms 


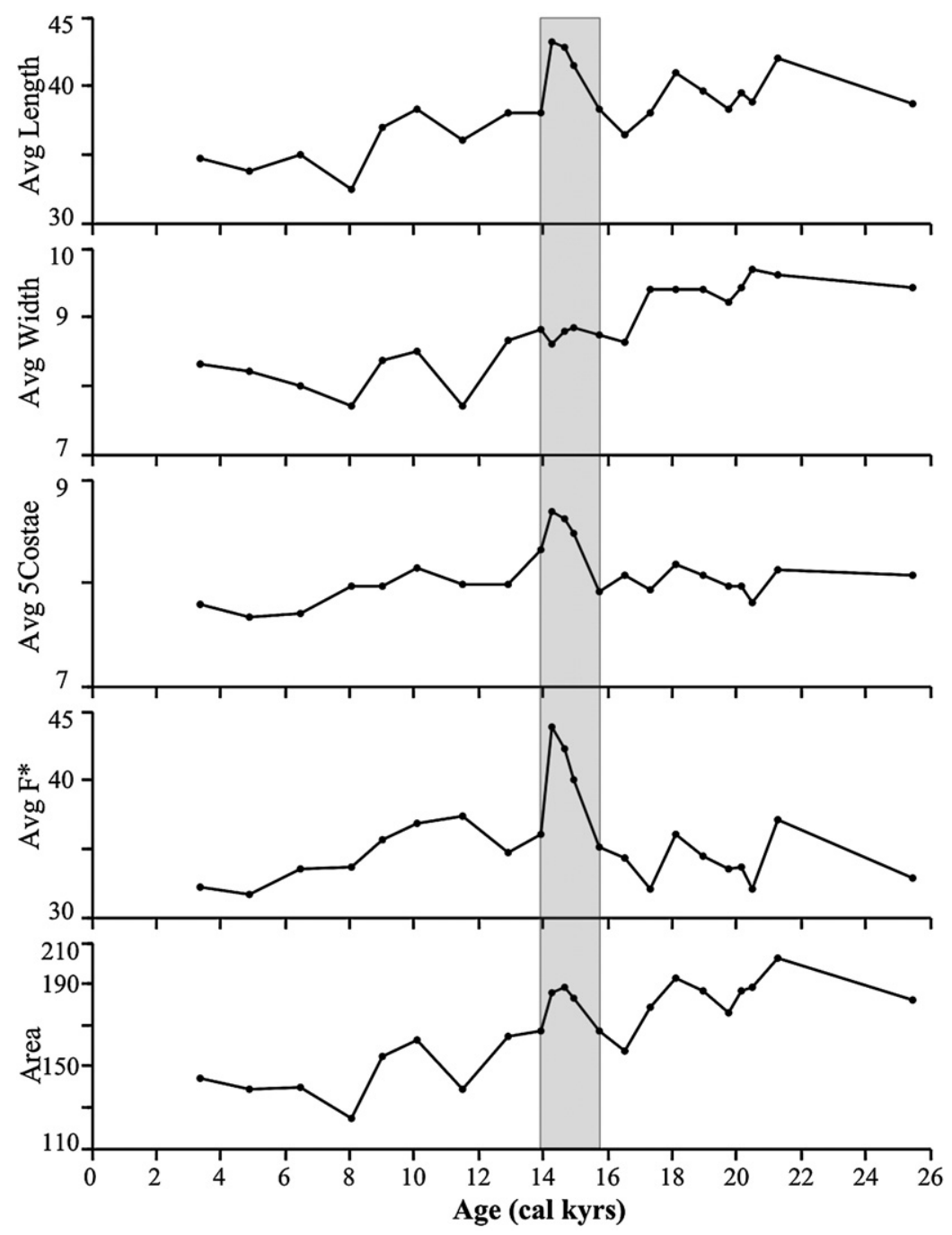

Fig. 5. Piston core PS1654-2 results. Time series at a location close to the Polar Front (piston core PS1654-2, $50^{\circ} 09^{\prime} .45 \mathrm{~S}, 05^{\circ} 43^{\prime} .35 \mathrm{E}$ ) for $F$. kerguelensis average valve length, width, length of five costae, $F^{*}$ and valve area. The $F$. kerguelensis shape shift interval discussed in the text is highlighted (light gray).

from the surface waters that will then sink to form AAIW and Antarctic Mode Waters. These water masses represent the main transport agent of nutrients to the thermocline, replenishing with nutrients, and thus fuelling, low latitude upwelling systems.

The main result of a previous study [10] on water samples from a Pacific Sector transect was the observation that the $F^{*}$ variable increases almost constantly from ca. $25 \mu \mathrm{m}$ (at $53^{\circ} \mathrm{S}$ ) to $37 \mu \mathrm{m}$ (maximum value at the Antarctic divergence, $68^{\circ} \mathrm{S}$ ).

Our results from the Pacific Sector, from roughly the same latitudes, but different sample sets (we used here surface sediment samples), match very well their observations, as we also documented an increase of $F^{*}$ from $25 \mu \mathrm{m}$ to $36 \mu \mathrm{m}$, and the presence of a maximum at $68^{\circ} \mathrm{S}$.
The general trend recognized by both studies is therefore that, as SST increases, $F^{*}$ decreases, i.e. longer and slimmer $F$. kerguelensis valves having fewer costae over $5 \mu \mathrm{m}$ are increasing towards cold waters, while broader, shorter $F$. kerguelensis with more costae are typical for warm waters.

Our study, however, reveals the presence of a distinct decrease in $F^{*}$ at higher latitudes, as well as a marked longitudinal variability. While the first observation pretty much invalidates the interpretation of $F^{*}$ in terms of SST alone, the strong longitudinal variability does not allow an extension of the Pacific Sector results to other Southern Ocean regions. As an example of this (Fig. 2E), one may observe the $F^{*}$ values along $57^{\circ} \mathrm{S}$ latitude in the different sectors, and 


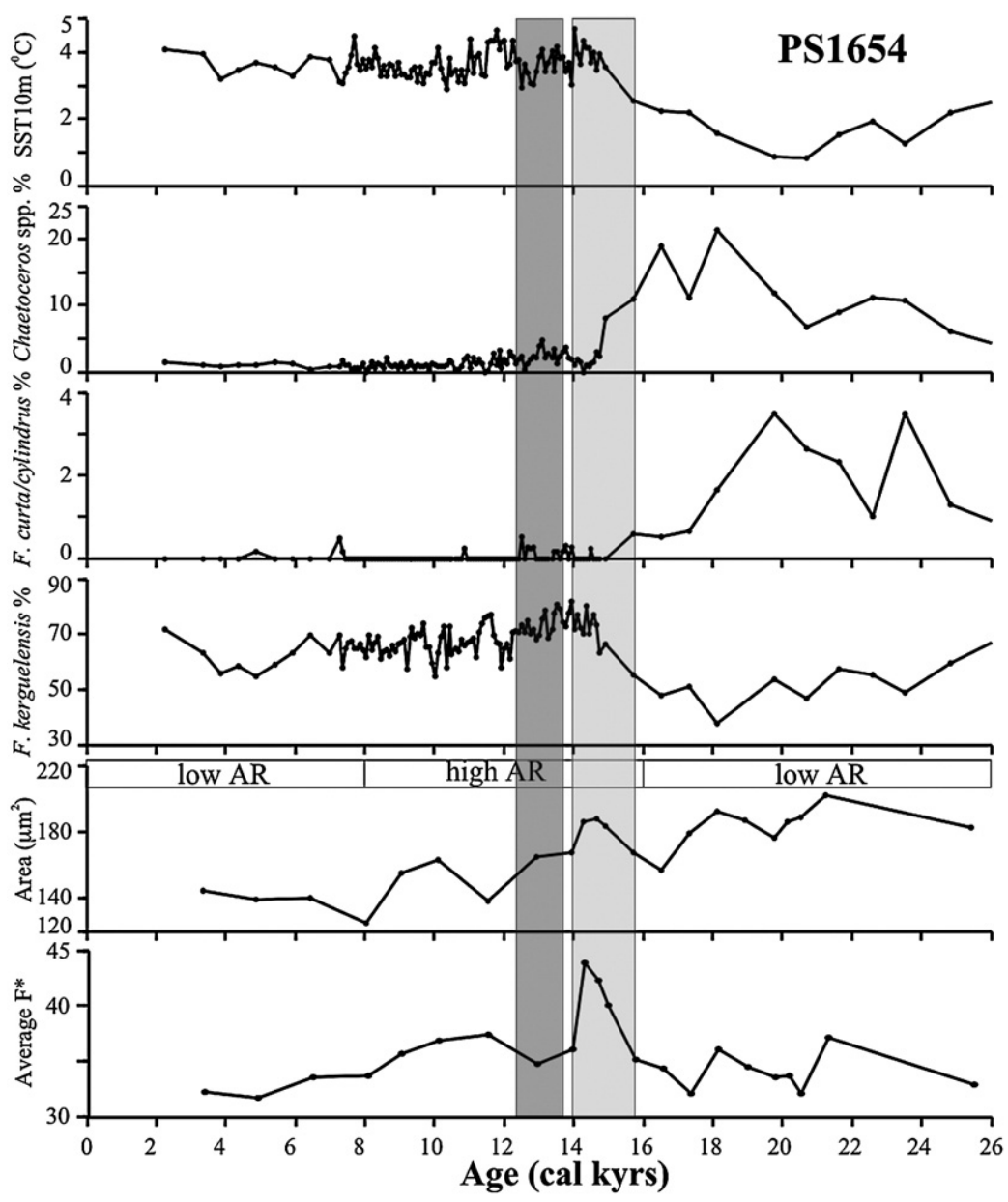

Fig. 6. Comparison of paleobiological proxies in piston core PS1654-2. Time series for SST, Chaetoceros spp., F. curta/cylindrus group, and F. kerguelensis relative abundances for piston core PS1654-2/ODP Site 1093 (data from [17]), and F. kerguelensis valve area and $F^{*}$ (piston core PS1654-2, this paper). A major meltwater event (dark gray), correlative to the ACR, and the $F$. kerguelensis shape shift interval (light gray) have been highlighted to facilitate comparison. Meltwater peaks at 14 and $11 \mathrm{kyr}$ BP have also been recognized close to the APF in another core (TN057-13) from the Atlantic Sector of the Southern Ocean [27]. The age scale [17] is in calendar years, with an 800 year reservoir correction. Intervals of higher and lower sediment accumulation rates have also been indicated.

notice how they (and therefore the general morphology of F. kerguelensis) are very different in the Pacific, Atlantic and Indian Sectors: intermediate $F^{*}$ values at $115^{\circ} \mathrm{W}$ contrast with low values at $90^{\circ} \mathrm{W}$, while they increase from $60^{\circ} \mathrm{W}$ to $40^{\circ} \mathrm{E}$, reaching a maximum in the Indian Sector.

Nonetheless, the length of five costae and $F^{*}$ (a linear combination of valve length, width, and length of five costae, representing a measure of the costae density "weighted" by the valve length and width) are better proxies for SST and/or salinity compared to the other morphometric variables (correlation coefficients ca. 0.5 to 0.6, see Appendix Table 1). Biplots of 5 costae length from all surface sediment samples versus both salinity and SST at the same locations (not shown) allow to recognize three "outliers". These three stations are the only ones in our dataset located to the north of the Subtropical Front and might represent a very special shape of $F$. kerguelensis compared to the rest of the Southern Ocean. Excluding these three stations from the regression, we obtain $r=0.77$ for the 5 costae length/SST biplot.

Our sediment trap study reveals how the seasonal variability in valve size, together with the lower diatom flux during the autumn/winter season, would suggest that most of the long-term signal, eventually stored in sediments, will be generated by the larger "spring/summer" F. kerguelensis: Higher diatom fluxes are recorded during the spring/summer (Fig. 4g) and correspond to larger sizes of $F$. kerguelensis (Fig. $4 \mathrm{a}-\mathrm{b}$ ). This result also underscores the strong linkage between higher fluxes of biogenic silica to the bottom [25], higher thorium-corrected accumulation rates [26], and larger 

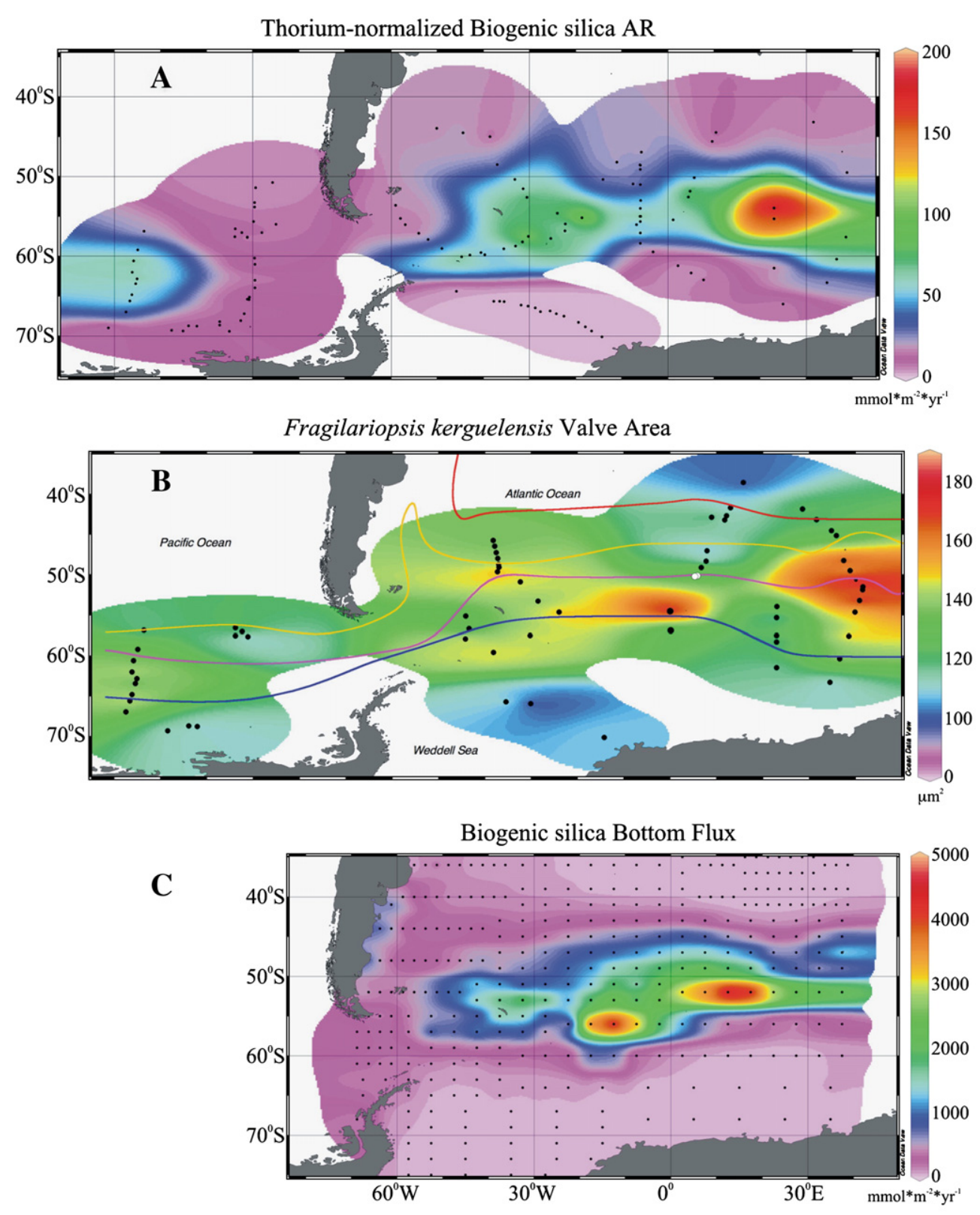

Fig. 7. Biogenic silica accumulation rates, bottom flux, and Fragilariopsis kerguelensis valve area. Comparison between: A) thorium-normalized accumulation rates of biogenic silica to the sediment [26], B) F. kerguelensis valve area (this study), C) biogenic silica bottom flux [25]. The sample coverage for the different maps is shown by the black dots, while oceanic frontal positions and maximum sea ice extent are according to [30]: Subtropical Front (STF, red), Subantarctic Front (SAF, yellow), Polar Front (APF, purple), winter sea ice edge (WSI, blue). The raw data from the above mentioned studies have been gridded with the OceanDataView software [22].

valves in F. kerguelensis, as observed in the geographic distribution of these variables (Fig. 7).

The average area $\left(124.6 \mu \mathrm{m}^{2}\right.$, horizontal dashed line in Fig. 4a-b, see also Table 2) for a coretop (PS1759-1) located in the immediate proximity of the sediment trap site indeed confirms this observation, as it is much closer to the spring/summer $\left(123.1 \mu^{2}\right)$ than to the autumn/winter $\left(106.3 \mu^{2}\right)$ average value. This size variability is most likely physiology/biology related, with $F$. kerguelensis valves being larger during bloom seasons, in response to higher available nutrient levels, and as protection against predators. The yearly averaged upper $\left(117.1 \mu^{2}\right)$ and lower $\left(120.1 \mu^{2}\right)$ trap valve area (Table 2) also compare relatively well to the "long term" coretop average size $\left(124.6 \mu \mathrm{m}^{2}\right)$. The latter is probably slightly skewed towards higher values, as it is 
more representative of the prevailing spring/summer conditions (larger F. kerguelensis, stronger export fluxes, better preservation efficiency in surface sediments) over the last few hundreds of years.

\subsection{Piston core PS1654-2: glacial to interglacial conditions}

The downcore record of morphology changes in $F$. kerguelensis displays three features:

1) a major reduction in valve size from glacial to interglacial.

The valve size during full glacial conditions (193.6 $\mu \mathrm{m}^{2}$ in average) is higher than what observed anywhere in the study area today, as the maximum valve area $\left(189.9 \mu \mathrm{m}^{2}\right)$ was documented in coretop PS2609-2, collected from the Polar Front in the Atlantic-Indian Sector of the Southern Ocean.

2) a very peculiar morphology with longer and larger valves having less densely spaced costae occurring during the latest part of the deglaciation, at ca. $14.5 \mathrm{ka}$; The average valve area for the three samples indicative of this time interval (see Table 3) is $191.0 \mu \mathrm{m}^{2}$, indicating how the average size of $F$. kerguelensis valves was almost back to full glacial values in the proximity of the Polar Front.

3) a minor discrepancy between the observed average interglacial size and the corresponding modern value.

The average valve area during full interglacial conditions (148.9 $\mu^{2}$, Figs. 5-6, Table 3) is ca. $25 \mu \mathrm{m}^{2}$ larger than the corresponding surface sediment average at the Polar Front $\left(124.6 \mu \mathrm{m}^{2}\right)$, and comparable to the highest values $\left(148\right.$ to $151 \mu^{2}$ ) recorded for the sediment trap samples, and associated to peak spring bloom conditions. This has two, possibly concurring, explanations:

a) a trend towards size reduction is present during the last $3 \mathrm{ka}$ (which have not been recovered in core PS1654-2);

b) the sediment core samples are representative of full spring bloom conditions (and associated increased diatom flux/preservation efficiency), with dissolution through the water column and at the sediment/water interface being able to remove, on centennial/millennial timescales, the documentation of weaker-/nonbloom conditions.

The first two points deserve much more attention, as they might have important implications for the paleo- ceanographic interpretation of the observed signal. The average width and length of $F$. kerguelensis display a clear pattern in the downcore record (Fig. 5), with higher values during the glacial and lower during the interglacial. As both variables change roughly in parallel, the average area does the same. The average length of five costae and the related variable $F *$ do not have this pattern of variation, as their glacial and Holocene values are quite similar. However, a shape shift in $F$. kerguelensis occurs at $14.5 \mathrm{ka}$, giving rise to a unique morphology: the $F *$ variable peaks, valve area slightly increases, the valves are the longest ever recorded but remain narrow, and the length of five costae also reaches maximum values. The $F$. kerguelensis specimens from this interval are therefore unusually long and with less dense costae than ever, and their occurrence suggests how very specific shapes of $F$. kerguelensis, in terms of $F * /$ costae density (possibly a measure of different valve robustness, resistance to stress, or degree of silicification) but also average valve area, occur over different parts of the Southern Ocean and/or the paleorecord. This specific morphology and the very sharp $F^{*}$ peak occur just prior to a meltwater event, and are the culminating point of a sequence of important paleoecological and paleoceanographical changes at the Antarctic Polar Front over last $25 \mathrm{ka}$, well recorded in core PS1654 (Fig. 6): during the last part of the glacial, at ca. $20 \mathrm{ka}$, sea ice starts to retreat (decrease in abundance of the diatoms F. curta and F. cylindrus), the general diatom assemblage switches suddenly, with Chaetoceros spp. being replaced by $F$. kerguelensis as the dominant diatom species over the interval between 18 and $14 \mathrm{ka}$. The peak in $F^{*}$ occurs just when this switch is almost complete, and prior to a marked meltwater event (dark gray column in Fig. 6).

The surface sediment dataset allows to provide an interpretation for this peak: under modern conditions, $F^{*}$ is anticorrelated (Appendix Table 1) to summer SST and salinity ( $r=-0.549$ and -0.407 , respectively), and directly correlated to the annual dissolved silicon and nitrate content of waters at $10 \mathrm{~m}$ depth $(r=0.535$ and $0.473)$. The $F^{*}$ peak at $14.5 \mathrm{ka}$ in core PS1654 occurs during a strong rise in SST towards maximum Holocene values (Fig. 6), which should push $F^{*}$ towards lower values, contrary to what observed. In order to compensate for this, either salinity (also anticorrelated to $F^{*}$ ) strongly decreases during this time interval, or dissolved silicon and/or nitrate increased markedly. As there is no evidence for lower salinity over this portion of the record (a meltwater peak occurs a few ka later), we favour the second hypothesis: a change in nutrient concentrations in surface waters. This is particularly alluring as iron-limited diatoms have been demonstrated 
$[4,5]$ to produce thicker valves, and to have higher $\mathrm{Si} / \mathrm{N}$ uptake ratios than under iron-replete conditions. As a consequence of these two observations, and remembering how higher $F^{*}$ values represent less robustly built diatoms (more widely spaced costae), a possible explanation is that a relief from iron-limitation, and additional availability of dissolved silicon in the water column took place at ca. 14-15 ka, and caused the peak in valve size and a distinctive, less robustly built morphology for this diatom (Fig. 6). In fact, this interval would represent the first opportunity for F. kerguelensis, since glacial times, to build longer valves with more widely spaced costae, as the underway deglaciation (sharp SST rise) causes sea ice to retreat (as documented by the drop in F. curta/cylindrus group). At the same time, Chaetoceros spp., a potential competitor for dissolved silicon usage, drops in abundance. As a consequence of all these ecosystem changes, $F$. kerguelensis strongly increases not only its relative abundance to ca. $70-80 \%$ at the Polar Front, but also its average valve size, resulting in the establishment of high opal accumulation rates starting from ca. $16 \mathrm{ka}$. These events occur just before the 12-14 ka interval (also highlighted in Fig. 6), for which the paleoclimatic record of core PS1654-2 [17] provides evidence for the occurrence, at ca. $50^{\circ} \mathrm{S}$, of several interesting paleoceanographic events. These events are quite distinct at a more southerly core location (ca. $53^{\circ} \mathrm{S}$, core PS2090/ODP Site 1094), but are still evident at the PS1654-2 core site (i.e. at the APF):

a) cooling of surface water temperatures during the deglaciation;

b) increase in the relative abundance of winter sea-ice extent indicator species (F. curta/cylindrus group) south of the Polar Front;

c) increase in the relative abundance of Chaetoceros spp., a possible meltwater proxy.

Meltwater peaks bracketing this time interval have also been recognized in the Atlantic Sector of the Southern Ocean [27] by $\delta^{18} \mathrm{O}_{\text {diatom }}$ at 14 and $11 \mathrm{kyr}$ BP in core TN057-13, sampled just south of the APF. These changes go in the opposite direction as what mentioned for the 14-15 ka interval, and thus result in a size (and, partly, relative abundance) reduction for F. kerguelensis. This may indicate changes in the diatom assemblage related to opposing life-strategies by F. kerguelensis and Chaetoceros spp. [16].

A similar difference in nutrient relative utilization has been demonstrated, for the Atlantic Sector of the Southern Ocean, by $\delta^{15} \mathrm{~N}$ (bulk sediment) and $\delta^{30} \mathrm{Si}$ (diatoms) isotopes [28,29] suggesting how, during glacials, dissolved silicon is used more efficiently compared to nitrate, while the opposite is true during interglacials. This would lead, south of the APF, to a tendency towards nitrate depletion during glacials (with preferential export, via intermediate waters, of dissolved silicon out of the Southern Ocean), and towards silicate depletion during interglacials (and a switch to nitrate export from the Southern Ocean). The consequences of this could be increased dissolved silicon availability and/ or lower $\mathrm{Si} / \mathrm{N}$ uptake ratios in diatoms (due to the relief from iron-limited conditions) during full glacial conditions, and thus diatoms may be able to build larger tests compared to interglacial conditions, a possible explanation for the observed larger sizes of F. kerguelensis during glacial intervals.

\section{Conclusions and perspectives}

The main outcome of this study is that valve areas in F. kerguelensis reach maximum values in the proximity of the APF, and can therefore be used to trace past positions of this belt of high biogenic silica export.

During glacial times, valves are even bigger than what one would expect by a comparison to oceanic zones displaying similar SST/nutrient conditions according to the recent situation, due to either the mentioned relief from silica-limitation during glacial times (i.e. possibility for $F$. kerguelensis to build even bigger valves than anywhere in this area today), or to the expansion of the "opal belt" and APF over the study site.

The observed size changes in F. kerguelensis between glacial and interglacial intervals therefore seem to confirm the conclusions derived from $\delta^{15} \mathrm{~N}$ and $\delta^{30} \mathrm{Si}$ isotopic studies, as this species has, close to the Polar Front, larger valve size during glacials (i.e. efficient dissolved silicon utilization, dissolved silicon-replete conditions, as observed at the APF today), and smaller valves during interglacials (i.e. inefficient utilization, tendency towards dissolved silicon limitation, as observed north of the APF today).

These results may be related to relief from ironlimitation at the APF, which probably allowed lower $\mathrm{Si} / \mathrm{N}$ uptake ratios for $F$. kerguelensis and/or access to higher dissolved silicon content in the upper water column, thus giving this species the opportunity to increase its relative abundance and average size. These observations moreover could lend strong support to the interpretation of diatom size variability in terms of higher/lower iron concentration and silica-repleted/ depleted conditions at the Polar Front, and the associated impact on diatom $\mathrm{Si} / \mathrm{N}$ uptake ratios. Larger 
F. kerguelensis valves occur during glacial intervals and strong sea-ice retreats (i.e. higher iron concentration, silica-replete conditions), while smaller valves are associated with lower dissolved silicon and iron concentrations during interglacials.

The link between iron-repleted/depleted conditions proposed here is of a speculative nature, as it is more based on logical inferences derived from our knowledge of the study area and of the main changes occurring over glacial/interglacial scales, than on direct dissolved iron measurements. To our knowledge, such an extensive dataset of dissolved iron measurements covering the study area is simply not available yet, and would greatly contribute towards clarifying the causal relationship between macro-/micro-nutrient levels and diatom valve size.

A first important step in this direction is a planned cruise where we will sample surface waters at hundreds of sites along a transect in the Southern Ocean, and analyze at the same time diatom morphometrics, $\mathrm{Si}$ and $\mathrm{N}$ isotopes, macronutrients and Fe content of seawater. This combination of paleobiological proxies with isotopic and macro-/micro-nutrient measurements will allow us to better understand the causal relationships between size variations in diatoms and their utilization of different nutrients.

While $F$. kerguelensis is such a dominant component of the diatom assemblages, and most likely the main silica carrier in the iron-limited Southern Ocean, other species (Thalassiosira lentiginosa, Thalassiotrix antarctica) may occasionally contribute significantly to the biogenic silica signature. In order to test their contribution, an integration of data from these species is desirable, and may refine the results presented in this study.

We are also currently developing methods to directly measure the volume of single diatom cells, which will help to quantify the relative contribution of different species to the total biogenic silica export, and thus get a better understanding of how changes in diatom assemblage composition may have affected silica export both in the geological past, and over a seasonal scale. Moreover, we intend to measure silicification for diatoms deposited both during glacial and interglacial intervals, to test whether or not the increase in valve area goes along with an increase in the amount of silica per valve.

\section{Acknowledgments}

Funded by the Deutsche Forschungsgemeinschaft as part of the DFG-Research Center "Ocean Margins" of the University of Bremen, no. RCOM0489. Thanks to Christina De La Rocha for her comments on an earlier draft, and to three anonymous reviewers, whose thoughtful comments greatly improved the quality of this manuscript. Part of the chlorophyll data used in this study were acquired as part of the NASA's Earth Science Enterprise. The algorithms were developed by the MODIS Science Teams. The data were processed by the MODIS Adaptive Processing System (MODAPS) and Goddard Distributed Active Archive Center (DAAC), and are archived and distributed by the Goddard DAAC.

\section{Appendix A. Supplementary data}

Supplementary data associated with this article can be found, in the online version, at doi:10.1016/j. eps1.2007.03.021.

\section{References}

[1] J.H. Martin, S.E. Fitzwater, Iron deficiency limits phytoplankton growth in the north-east Pacific subarctic, Nature 331 (1988) 341-343.

[2] J.H. Martin, M. Gordon, S. Fitzwater, Iron in Antarctic waters, Nature 345 (1990) 156-158.

[3] K.H. Coale, K.S. Johnson, S.E. Fitzwater, R.M. Gordon, S. Tanner, F.P. Chavez, L. Ferioli, C. Sakamoto, P. Rogers, F. Millero, P. Steinberg, P. Nightingale, D. Cooper, W.P. Cochlan, M.R. Landry, J. Constantinou, G. Rollwagen, A. Trasvina, R. Kudela, A massive phytoplankton bloom induced by an ecosystem-scale iron fertilization experiment in the equatorial Pacific Ocean, Nature 383 (1996) 495-501.

[4] S. Takeda, Influence of iron availability on nutrient consumption ratio of diatoms in oceanic waters, Nature 393 (1998) 774-777.

[5] D.A. Hutchins, K.W. Bruland, Iron-limited diatom growth and Si:N uptake ratios in a coastal upwelling regime, Nature 393 (1998) 561-564.

[6] H.J.W. De Baar, A.G.J. Buma, R.F. Nolting, G.C. Cadée, G. Jacques, P.J. Tréguer, On iron limitation of the Southern Ocean: experimental observations in the Weddell and Scotia Seas, Mar. Ecol. Prog. Ser. 65 (1990) 105-122.

[7] J.H. Martin, K.H. Coale, K.S. Johnson, S.E. Fitzwater, R.M. Gordon, S.J. Tanner, C.N. Hunter, V.A. Elrod, J.L. Nowicki, T.L. Coley, R.T. Barber, S. Lindley, A.J. Watson, K. Van Scoy, C.S. Law, M.I. Liddicoat, R. Ling, T. Stanton, J. Stockel, C. Collins, A. Anderson, R. Bidgare, M. Ondrusek, M. Latasa, F.J. Millero, K. Lee, W. Yao, J.Z. Zhang, G. Friederich, C. Sakamoto, F. Chavez, K. Buck, Z. Kolber, R. Greene, P. Falkowski, S.W. Chisholm, F. Hoge, R. Swift, J. Yungel, S. Turner, P. Nightingale, A. Hatton, P. Liss, N.W. Tindale, Testing the iron hypothesis in ecosystems of the equatorial Pacific Ocean, Nature 371 (1994) 123-129.

[8] J.L. Sarmiento, N. Gruber, M.A. Brzezinski, J.P. Dunne, Highlatitude controls of thermocline nutrients and low latitude biological productivity, Nature 427 (2004) 56-60.

[9] D.M. Nelson, P. Trèguer, M.A. Brzezinski, A. Leynaert, B. Quèguiner, Production and dissolution of biogenic silica in the 
ocean: revised global estimates, comparison with regional data and relationship to biogenic sedimentation, Glob. Biogeochem. Cycles 9 (1995) 359-372.

[10] J. Fenner, H.J. Schrader, H. Wienigk, Diatom phytoplankton studies in the southern Pacific Ocean, composition and correlation to the Antarctic Convergence and its paleoecological significance, in: C.D. Hollister, C. Craddock, et al., (Eds.), Initial Reports of the Deep Sea Drilling Project, vol. 35, U.S. Govt. Printing Office, Washington, 1976, pp. 757-813.

[11] U. Zielinski, R. Gersonde, Diatom distribution in Southern Ocean surface sediments (Atlantic sector): implications for paleoenvironmental reconstructions, Palaeogeogr. Palaeoclimatol. Palaeoecol. 129 (1997) 213-250.

[12] J.G. Donahue, Diatoms as Quaternary biostratigraphic and paleoclimatic indicators in high latitudes of the Pacific Ocean, Ph.D. thesis, Columbia University, 1970.

[13] L.H. Burckle, J. Cirilli, Origin of diatom ooze belt in the Southern Ocean: implications for late Quaternary paleoceanography, Micropaleontology 33 (1987) 82-86.

[14] C.E. Hamm, R. Merkel, O. Springer, P. Jurkojc, C. Maier, K. Prechtel, V. Smetacek, Architecture and material properties of diatom shells provide effective mechanical protection, Nature 421 (2003) 841-843.

[15] V. Smetacek, P. Assmy, J. Henjes, The role of grazing in structuring Southern Ocean pelagic ecosystems and biogeochemical cycles, Antarct. Sci. 16 (4) (2004) 541-558.

[16] A. Abelmann, R. Gersonde, G. Cortese, G. Kuhn, V. Smetacek, Extensive phytoplankton blooms in the Atlantic sector of the glacial Southern Ocean, Paleoceanography 21 (2006) PA1013, doi:10.1029/2005PA001199.

[17] C. Bianchi, R. Gersonde, Climate evolution at the last deglaciation: the role of the Southern Ocean, Earth Planet. Sci. Lett. 228 (2004) 407-424.

[18] K.H. Coale, K.S. Johnson, F.P. Chavez, K.O. Buesseler, R.T. Barber, M.A. Brzezinski, et al., Southern Ocean iron enrichment experiment: carbon cycling in high- and low-Si waters, Science 304 (5669) (2004) 408-414.

[19] G. Fischer, R. Gersonde, G. Wefer, Organic carbon, biogenic silica and diatom fluxes in the marginal winter sea-ice zone and in the Polar Front Region: interannual variations and differences in composition, Deep-Sea Res. II 49 (2002) 1721-1745.

[20] R. Gersonde, U. Zielinski, The reconstruction of late Quaternary Antarctic sea-ice distribution: the use of diatoms as a proxy for seaice, Palaeogeogr. Palaeoclimatol. Palaeoecol. 162 (2000) 263-286.

[21] G.R. Hasle, Nomenclatural notes on marine planktonic diatoms, The family Bacillariaceae, Nova Hedwigia, Beiheft, vol. 106, 1993, pp. 315-321.

[22] R. Schlitzer, Ocean Data View, http://odv.awi-bremerhaven.de2005.

[23] M.E. Conkright, R.A. Locarnini, H.E. Garcia, T.D. O'Brien, T.P. Boyer, C. Stephens, J.I. Antonov, World Ocean Atlas 2001: objective analyses, data statistics, and figures, CD-ROM Documentation, National Oceanographic Data Center, Silver Spring, MD, 2002, p. 17

[24] V. Makarenkov, P. Legendre, Non linear redundancy analysis and canonical correspondence analysis based on polynomial regression, Ecology 83 (2002) 1146-1161.

[25] R. Usbeck, M. Rutgers van der Loeff, M. Hoppema, R. Schlitzer, Shallow remineralization in the Weddell Gyre, Geochem. Geophys. Geosyst. 3 (1) (2002), doi:10.1029/2001GC000182.

[26] W. Geibert, M.M. Rutgers van der Loeff, R. Usbeck, R. Gersonde, G. Kuhn, J. Seeberg-Elverfeldt, Quantifying the opal belt in the Atlantic and southeast Pacific sector of the Southern Ocean by means of ${ }^{230} \mathrm{Th}$ normalization, Glob. Biogeochem. Cycles 19 (2005) GB4001, doi:10.1029/2005GB002465.

[27] A. Shemesh, D. Hodell, X. Crosta, S. Kanfoush, C. Charles, T. Guilderson, Sequence of events during the last deglaciation in Southern Ocean sediments and Antarctic ice cores, Paleoceanography 17 (4) (2002) 1056, doi:10.1029/2000PA000599.

[28] C.L. De La Rocha, M.A. Brzezinski, M.J. DeNiro, A. Shemesh, Silicon-isotope composition of diatoms as an indicator of past oceanic change, Nature 395 (1998) 680-683.

[29] M.A. Brzezinski, C.J. Pride, V.M. Franck, D.M. Sigman, J.L. Sarmiento, K. Matsumoto, N. Gruber, G.H. Rau, K.H. Coale, A switch from $\mathrm{Si}(\mathrm{OH})_{4}$ to $\mathrm{NO}^{3-}$ depletion in the glacial Southern Ocean, Geophys. Res. Lett. 29 (2002), doi:10.1029/2001GL014349.

[30] I.M. Belkin, A.L. Gordon, Southern Ocean fronts from the Greenwich meridian to Tasmania, J. Geophys. Res. 101 (C2) (1996) 3675-3696. 